\section{GLRT Subspace Detection for Range and Doppler Distributed Targets}

\author{
NICOLAS BON \\ ALI KHENCHAF, Member, IEEE \\ ENSIETA \\ France \\ RENÉ GARELLO, Fellow, IEEE \\ ENST \\ France
}

A generalized likelihood ratio test (GLRT) is derived for adaptive detection of range and Doppler-distributed targets. The clutter is modeled as a spherically invariant random process (SIRP) and its texture component is range dependent (heterogeneous clutter). We suppose here that the speckle component covariance matrix is known or estimated thanks to a secondary data set. Thus, unknown parameters to be estimated are local texture values, the complex amplitudes and Doppler frequencies of all scattering centers. To do so, we use superresolution methods. The proposed detector assumes a priori knowledge on the spatial distribution of the target and has the precious property of having a constant false alarm rate (CFAR) with the assumption of a known speckle covariance matrix or by the use of frequency agility.

This work was supported by a grant from Délégation Générale de l'Armement (DGA).

Authors' addresses: N. Bon, 10, avenue de la Iere D.F.L. CS 93801, 29238 Brest, Cedex 3, France, E-mail: (nicolas.bon@gmail.com); A. Khenchaf, ENSIETA, Laboratoire E312-EA3876, 2 Rue Francois Verny, Brest 29806, France; R. Garello, ENST Bretagne, Brest, France.

\section{INTRODUCTION}

Pulse compression techniques enable radars to achieve a high range resolution, thus resolving the target into a number of scattering centers. The range resolution is inversely proportional to the transmitted bandwidth of the radar [1]. High-range resolution radars (HRRs) are then able to form the range target response (range profile) or to form the bidimensional target response by radar imagering techniques (synthetic aperture radar/inverse synthetic aperture radar (SAR/ISAR)). Current radars scheme is to first detect the target in a low or medium resolution (typically $30 \mathrm{~m}$ ) and then to form the target response.

In the last few years, many works have been led on high-range resolution detectors, which can be seen as matched filtering to target response. These detectors can be based on different signal and clutter models resulting in various expressions and performances. Van Trees [2] examined several detectors designed for range-spread targets. These techniques and others were further examined by Steiner [3]. Hughes [4] compared the performances of two different range-distributed detectors on an HRR: a 1 out of $M$ detector and a noncoherent detector that integrates the different range cells. It was shown that when the target is distributed over just a few cells, the 1 out of $\mathrm{M}$ detector gives the best results whereas when the target is distributed over many cells, the noncoherent integrator gives the best performances. Rose [5] obtained similar results and Gerlach, et al. [6] compared the performances of the 1 out of $\mathrm{M}$ detector with a noncoherent generalized likelihood ratio test (GLRT) detector where the a priori scatterers distribution is known. In [7], [8], Farina, et al. examined more specific aspects concerning the ultrawideband (UWB) detection and identification. They derived a likelihood ratio test (LRT) under the hypothesis that the target is a zero-mean Gaussian process of known covariance matrix. The LRT was a quadratic form with respect to the received signal vector and a function of the target and clutter covariance matrix. Distributed target detectors in white Gaussian noise and with the constant false alarm rate (CFAR) property have been proposed in [9], [10], [11]. They were based on the GLRT and the returns of different range cells were modeled as independent and identically distributed Gaussian vectors of unknown covariance matrix. In addition, a secondary data set is present to estimate the clutter covariance matrix which is supposed to be the same as for the primary data. The same approach is adopted by Kelly in [12]. The considered configurations then assume a homogeneous background. In [13], Gerlach and Steiner derived a modified GLRT for the adaptive detection of range-spread targets. The target amplitudes and clutter covariance matrix are supposed to be unknown 
but the strategy does not resort to secondary data. The distribution of the modified GLRT, under the clutter-only hypothesis, depends on the clutter covariance matrix and thus has not the quality of being a CFAR detector. However, the authors show that their detector can be CFAR-bounded. They proposed another CFAR-bounded detector in [14] designed for range-spread and Doppler-shifted targets in disturbance of unknown but structured covariance matrix. More recently, Conte, De Maio, and Ricci [15] proposed a GLRT for range-spread targets in Gaussian clutter with unknown covariance matrix. They also resort to secondary data, free of signal components, which power can be different from primary data. The power ratio is then maximum likelihood (ML)-estimated. They proposed two detectors corresponding to two different cases where the covariance matrix is totally unknown or where its structure is known. The detectors take the form of the GAMF (Gaussian adaptive matched filter), a generalization to distributed targets of the AMF proposed by Robey, et al. in [16], and the GASD (generalized adaptive subspace detector). These detectors have the CFAR quality. Jin and Friedlander [17] have also studied the case of a target modeled as a Gaussian vector in Gaussian disturbance with unknown covariance matrices estimated thanks to a secondary data set. The detector, based on subspace decomposition, is CFAR.

In all previous cited detectors, the clutter distribution was supposed to be Gaussian. However, in HRR mode, clutter statistics cannot be modeled as Gaussian random process anymore due to the observation of spikes. The distribution is usually modeled as a compound Gaussian vector and more precisely, as a spherically invariant random vector (SIRV) [18]. The clutter vector is then the product of two components: 1) a rapid fluctuation component, so-called speckle, which decorellation time is about $10 \mathrm{~ms}$ in the case of sea clutter and which can be entirely decorrelated with the use of frequency agility, and 2) a slow fluctuation modulating component so-called texture that exhibits a much longer decorrelation [19] time and which is not affected by frequency agility. Gerlach [20] and Conte, et al. [21] extended their CFAR detectors to non-Gaussian clutter modeled as a SIRV. The texture component is then ML-estimated in each range cell.

All these works show that a properly designed detector for range-spread targets on high-resolution radars gives best performances with respect to a low or medium resolution radar where the target is entirely contained in one range cell. In fact, HRRs enable to give a sampled response of the target so that the backscattered signal is not a simple delayed replica of the transmitted waveform but its convolution with the target response. As Farina, et al. described in [22], [23], [24], high-range resolution detectors can be seen as matched detectors to the target response and to the transmitted waveform. The performance improvement depends upon the fact that increasing the range resolution of the radar reduces the clutter energy in each range cell. Moreover, resolved scatterers are less fluctuating than a point target fully contained in one range cell. Indeed, in low-range resolution, the target response is the vectorial sum of its different scatterers' contributions which entails great fluctuations. Such targets are often represented as Swerling [25] or Nakagami's [26] models.

We propose here to extend the previously derived range-distributed detector to the bidimensional case. The detector is then designed for range and Doppler distributed target in non-Gaussian clutter. We then construct a detector matched to the bidimensional target response in range and Doppler frequency. Resolving the target on the Doppler axis enables to reduce the fluctuation of the scatterers with respect to a detector designed for range-only distributed targets. Otherwise, whereas the monodimensional response of the target may be seen as its range profile, estimating the $2 \mathrm{D}$ response of the target may be done with SAR or ISAR imaging techniques providing useful and additional information for a further classification step. This paper is organized as follows. In Section II, the problem statement is formulated and the clutter and signal models are described. The GLRT is derived in Section III, the CFAR property and the false alarm probability are discussed in Section IV and Section V is devoted to several results of our detector on synthetic data.

\section{PROBLEM STATEMENT AND SIGNAL MODEL}

We consider a signal at the output of the sensor composed of $N$ samples, corresponding to $N$ successive pulses of the radar, in each range cell. Thus, the measured signal is written $\mathbf{z}_{r}=$ $\left(z_{r}(0), z_{r}(1), \ldots, z_{r}(N-1)\right)^{t}$ and $\mathbf{Z}=\left[\mathbf{z}_{1}, \mathbf{z}_{2}, \ldots, \mathbf{z}_{L}\right]$ where the subscript $r$ refers to the range cell number.

We assume that the target to detect is spatially distributed over $L$ range cells. In a realistic scenario, this assumption cannot be made anymore. The estimation of $L$ is not tackled here and is left for future work. It may be based, for example, on an ML scheme. However, it is worth mentioning that Section V presents results concerning the influence of a mismatched length between the model and the true value.

The disturbance is only constituted of clutter. Here, we consider a clutter-dominant scenario so the influence of thermal noise may be neglected. This situation occurs for example in short-range detection schemes. The influence of thermal noise on the detector performances is tackled in Section V. $\mathbf{x}_{r}=\left(x_{r}(0), x_{r}(1), \ldots, x_{r}(N-1)\right)^{t}$ denotes 
the signal echoed by the target in range cell $r$ and $\mathbf{c}_{r}=\left(c_{r}(0), c_{r}(1), \ldots, c_{r}(N-1)\right)^{t}$ is the clutter vector. The azimuthal extension of the target is supposed very small with respect to the azimuthal beamwidth (for example the beamwidth of a radar antenna with aperture of $3.5 \mathrm{deg}$, is about $1 \mathrm{~km}$ at $10 \mathrm{nmi}$ ). As a consequence, target and clutter are present in each range cell and thus, the detection strategy is to test two different hypotheses which can be formulated as follows:

$$
\begin{aligned}
& H_{0}: \quad \mathbf{z}_{r}=\mathbf{c}_{r}, \quad r=1 \ldots L \\
& H_{1}: \quad \mathbf{z}_{r}=\mathbf{x}_{r}+\mathbf{c}_{r}, \quad r=1 \ldots L .
\end{aligned}
$$

The measured signals are supposed to be independent between each range cell. The $H_{0}$ hypothesis corresponds to only the presence of clutter and the $H_{1}$ hypothesis to the presence of target embedded in clutter.

\section{A. Clutter Subspace}

The clutter vector $\mathbf{c}_{r}=\sqrt{\tau_{r}} \mathbf{s}_{r}$ is modeled as a SIRV such that $\mathbf{s}_{r} \sim \mathcal{C N}(\mathbf{0}, \mathbf{M}), r=1 \ldots L$. $\mathbf{s}_{r}$ is commonly named the speckle component and in the case of sea clutter, its decorrelation time is about $10 \mathrm{~ms}$ [19] and is totaly decorrelated with frequency agility. In that case, the clutter covariance matrix is then identity: $\mathbf{M}=\mathbf{I}$. $\tau_{r}$, the so-called texture, is a real positive random process and exhibits a greater decorrelation time and is not affected by frequency agility. It is then assumed that the texture component in each range cell is constant over the $N$ pulses. This representation of SIRV is widely used to model the radar clutter $[19,18,20,27]$. The multivariate distribution of the clutter vector is given conditionally to the texture by

$$
p_{\mathbf{c}_{r} \mid \tau_{r}}\left(\mathbf{c}_{r} \mid \tau_{r}\right)=\frac{1}{\left(\pi \tau_{r}\right)^{N} \operatorname{det} \mathbf{M}} \exp \left(-\frac{\mathbf{c}_{r}^{H} \mathbf{M}^{-1} \mathbf{c}_{r}}{\tau_{r}}\right) .
$$

In this work, we assume the clutter covariance matrix $\mathbf{M}=E\left\{\mathbf{s}_{r} \mathbf{s}_{r}^{H}\right\}$ is known and normalized so that $[\mathbf{M}]_{i i}=$ $1, i=1 \ldots N$. Nevertheless, its estimation is tackled in Section $\mathrm{V}$ to evaluate the influence of thermal noise and of the size of secondary data. Indeed, in a realistic scenario, $\mathbf{M}$ must be estimated from secondary data as Gini, et al. [28-30], Pulsone and Raghavan [31] and Rangaswamy, et al. [32, 33] described for point targets in non-Gaussian disturbance. Conte, De Maio, and Ricci [15] also resort to secondary data to estimate the clutter covariance matrix for their range-distributed target detector. The loss induced by the lack of the knowledge of the matrix is investigated for Swerling I targets by Conte, Lops, and Ricci in [34] and by Gini, et al. [35-37]. Finally, the selection and cleaning of secondary data is studied by Conte in [38].

\section{B. Signal Subspace}

When the wavelength of the transmitted signal is small with respect to the target dimensions, the geometric theory of diffraction (GTD) [39] and its uniform version [40] applies and the backscattered field can then be seen as the sum of the different scatterers contributions of the target. Measures show that the radar properties of a lot of targets, such as planes, ships, etc., are well modeled considering that they are backscattered from few isolated target points. Furthermore, the isolated reflections are representative of the physical parameters of the targets [41-43].

We then model the signal vector echoed by the target, which we note $\mathbf{x}_{r}=\left(x_{r}(0), x_{r}(1), \ldots, x_{r}(N-1)\right)^{t}$, in each range cell $r$, as a sum of the contribution of $p_{r}$ scatterers, so that

$$
x_{r}(n)=\sum_{k=1}^{p_{r}} a_{r, k} \exp \left(j \phi_{r, k}(n)\right), \quad n=0 \ldots N-1 .
$$

Thus, the signal in each range cell can be expressed in a matrix formulation as

$$
\mathbf{x}_{r}=\mathbf{E}_{r} \mathbf{a}_{r}
$$

where $\mathbf{a}_{r}=\left(a_{r, 1}, a_{r, 2}, \ldots, a_{r, p_{r}}\right)^{t}$ is the vector of the complex amplitudes reflected by the scatterers. In the following development, we derive two GLRT detectors based on different models of $\mathbf{a}_{r}$. The first one is based on the hypothesis that $\mathbf{a}_{r}$ is a Gaussian random vector so that $\mathbf{a}_{r} \sim \mathcal{C N}\left(\mathbf{0}, \Sigma_{r}\right)$. The second detector is based on the assumption that we have no a priori knowledge about the distribution of $\mathbf{a}_{r}$ which is then modeled as a deterministic unknown vector. In that second case, the signal vector $\mathbf{x}_{r}$ is consequently a zero-mean complex Gaussian vector of covariance matrix $\mathbf{E}_{r} \Sigma_{r} \mathbf{E}_{r}^{H}$. The signal model is equivalent to the so-called Gaussian linear model. As explained in [27], two extreme cases are represented by Swerling I target signals for $p_{r}=1$ and Swerling II target signals for $p_{r}=N$. This signal model has been often used in radar detection problems but also in array processing scenarios, see [27] for references. The steering matrix $\mathbf{E}_{r}$ is expressed as

$$
\mathbf{E}_{r}=\left(\begin{array}{cccc}
1 & 1 & \cdots & 1 \\
e^{j \phi_{r, 1}(1)} & e^{j \phi_{r, 2}(1)} & \cdots & e^{j \phi_{r, p r}(1)} \\
\vdots & & & \vdots \\
e^{j \phi_{r, 1}(N-1)} & e^{j \phi_{r, 2}(N-1)} & \cdots & e^{j \phi_{r, p r}(N-1)}
\end{array}\right) .
$$

It is assumed that the target motion is slow enough or that the observation time is short so that the phase variation is linear:

$$
\phi_{r, k}(n)=2 \pi f_{r, k} n, \quad n=0 \ldots N-1
$$


and we assume the number of scatterers $p_{r}$ per range cell is lower than the number of integrated pulses $N$, i.e., $p_{r}<N, r=1 \ldots L$ and is constant during the $N$ pulses.

With these definitions, the measured signal distribution in each range cell is distributed as $\mathbf{z}_{r \mid \tau, H_{1}} \sim \mathcal{C N}\left(\mathbf{E}_{r} \mathbf{a}_{r}, \tau_{r} \mathbf{M}\right)$ in the case of an unknown deterministic $\mathbf{a}_{r}$ and as $\mathbf{z}_{r \mid \tau, H_{1}} \sim \mathcal{C N}\left(\mathbf{0}, \mathbf{E}_{r} \boldsymbol{\Sigma}_{r} \mathbf{E}_{r}^{H}+\tau_{r} \mathbf{M}\right)$ otherwise. For the following developments, it is useful to consider a different form of the signal vector so that

$$
\mathbf{x}_{r}=\mathbf{U}_{r} \mathbf{b}_{r}, \quad r=1 \ldots L
$$

where, taking the singular value decomposition, $\mathbf{E}_{r}=$ $\mathbf{U}_{r} \mathbf{S}_{r} \mathbf{V}_{r}^{H}, r=1 \ldots L, \mathbf{U}_{r}$ is the $N \times p_{r}$ unitary matrix of left singular vectors, $\mathbf{S}_{r}$ is the $p_{r} \times p_{r}$ diagonal matrix of non-zero singular values, and $\mathbf{V}_{r}^{H}$ is the $p_{r} \times p_{r}$ diagonal unitary matrix of right singular vectors. $\mathbf{b}_{r}$ is either an unknown deterministic vector or a zero-mean complex Gaussian vector of covariance matrix $\mathbf{R}_{\mathbf{b}_{r}}$.

\section{GLRT DERIVATION}

\section{A. Optimal Detector and GLRT}

We derive two different GLRT detectors based on two different hypotheses about the complex amplitude vectors $\mathbf{a}_{r}, r=1 \ldots L$. The first detector considers unknown deterministic $\mathbf{a}_{r}, r=1 \ldots L$ and is named deterministic scatterer model GLRT (DSM-GLRT). The second detector assumes $\mathbf{a}_{r} \sim \mathcal{C N}\left(\mathbf{0}, \Sigma_{r}\right), r=$ $1 \ldots L$ and is referred to as the Gaussian scatterer model GLRT (GSM-GLRT).

Considering the independence hypothesis of the range cells, conditionally to the values of the texture component, the scatterers' amplitudes and the steering matrices, the joint density under $H_{1}$ is, respectively, for DSM-GLRT detector:

$$
\begin{aligned}
p_{\mathbf{z}_{1: L} \mid \tau_{1: L}, \mathbf{b}_{1: L}, \mathbf{U}_{1: L}, \mathbf{M}, H_{1}}\left(\mathbf{z}_{1: L} \mid \tau_{1: L}, \mathbf{b}_{1: L}, \mathbf{U}_{1: L}, H_{1}\right) \\
=\prod_{r=1}^{L} \frac{\exp \left(-\left(\mathbf{z}_{r}-\mathbf{U}_{r} \mathbf{b}_{r}\right)^{H} \mathbf{M}^{-1}\left(\mathbf{z}_{r}-\mathbf{U}_{r} \mathbf{b}_{r}\right) / \tau_{r}\right)}{\left(\pi \tau_{r}\right)^{N} \operatorname{det}(\mathbf{M})}
\end{aligned}
$$

and for the GSM-GLRT detector:

$$
\begin{gathered}
p_{\mathbf{z}_{1: L} \mid \tau_{1: L}, \mathbf{b}_{1: L}, \mathbf{U}_{1: L}, \mathbf{M}, H_{1}}\left(\mathbf{z}_{1: L} \mid \tau_{1: L}, \mathbf{b}_{1: L}, \mathbf{U}_{1: L}, H_{1}\right) \\
=\prod_{r=1}^{L} \frac{1}{\pi^{N} \operatorname{det}\left(\mathbf{B}_{r}\right)} \exp \left(-\mathbf{z}_{r}^{H} \mathbf{B}_{r}^{-1} \mathbf{z}_{r}\right)
\end{gathered}
$$

where $\mathbf{B}_{r}=\mathbf{U}_{r} \mathbf{R}_{\mathbf{b}_{r}} \mathbf{U}_{r}^{H}+\tau_{r} \mathbf{M}$ is the covariance matrix of the measured vector. For both DSM and GSM GLRTs, under $H_{0}$ :

$$
\begin{aligned}
p_{\mathbf{z}_{1: L} \mid \tau_{1: L}, \mathbf{M}, H_{0}}\left(\mathbf{z}_{1: L} \mid \tau_{1: L}, H_{0}\right) & \\
= & \prod_{r=1}^{L} \frac{1}{\tau_{r}^{N} \pi^{N} \operatorname{det}(\mathbf{M})} \exp \left(-\frac{\mathbf{z}_{r}^{H} \mathbf{M}^{-1} \mathbf{z}_{r}}{\tau_{r}}\right) .
\end{aligned}
$$

According to the Neyman-Pearson (NP) criterion, the optimal detector is the LRT, which can be obtained by averaging with respect to the texture components $\tau_{r}$, $r=1 \ldots L$, and the signal components so that

$$
\begin{aligned}
\Lambda_{\mathrm{NP}}\left(\mathbf{z}_{1: L}\right) & =\frac{E_{\tau_{1: L}}\left\{p_{\mathbf{z}_{1: L} \mid \tau_{1: L}, H_{1}}\left(\mathbf{z}_{1: L} \mid \tau_{1: L}, H_{1}\right)\right\}}{E_{\tau_{1: L}}\left\{p_{\mathbf{z}_{1: L} \mid \tau_{1: L}, H_{0}}\left(\mathbf{z}_{1: L} \mid \tau_{1: L}, H_{0}\right)\right\}} \\
& =\frac{\prod_{r=1}^{L} \int_{0}^{\infty} p_{\mathbf{z}_{r} \mid \tau_{r}, H_{1}}\left(\mathbf{z}_{r} \mid \tau_{r}, H_{1}\right) p_{\tau_{r}}\left(\tau_{r}\right) d \tau_{r}}{\prod_{r=1}^{L} \int_{0}^{\infty} p_{\mathbf{z}_{r} \mid \tau_{r}, H_{0}}\left(\mathbf{z}_{r} \mid \tau_{r}, H_{0}\right) p_{\tau_{r}}\left(\tau_{r}\right) d \tau_{r}} .
\end{aligned}
$$

For notation convenience, the averaging over the signal components $\mathbf{U}_{r}$ and $\mathbf{a}_{r}, r=1 \ldots L$ are not represented. We notice in (11) that the evaluation of the NP detector needs a heavy computational integration along the underlying mixing distribution. For this reason and because we do not know a priori the distribution $p_{\tau}(\tau)$ of the texture component, we resort to a suboptimum approach based on the GLRT where the unknown texture component is modeled as a deterministic parameter and replaced in the GLRT with its ML-estimate.

The first strategy (DSM) assumes that the multivariate distribution of the scatterers complex amplitude vectors $\mathbf{b}_{r}, r=1 \ldots L$ is not a priori known. As for the texture component we can then model it as a deterministic parameter and replace it with its ML-estimate. This strategy, originally proposed in [44] and further investigated in [45], is adopted for the distributed detectors proposed in [14], [15], [20], [21]. With this strategy, the GLRT is then expressed as

$$
\begin{aligned}
& \Lambda_{\text {DSM-GLRT }}\left(\mathbf{z}_{1: L}\right) \\
& =\max _{\mathbf{U}_{1: L}, \mathbf{b}_{1: L}, \tau_{1: L}} \Lambda\left(\mathbf{z}_{1: L} \mid \mathbf{U}_{1: L}, \mathbf{b}_{1: L}, \tau_{1: L}\right) \\
& =\Lambda\left(\mathbf{z}_{1: L} \mid \hat{\mathbf{U}}_{1: L}, \hat{\mathbf{b}}_{1: L}, \hat{\tau}_{1: L}\right) \\
& =\frac{p_{\mathbf{z}_{1: L} \mid H_{0}}\left(\mathbf{z}_{1: L}-\hat{\mathbf{U}}_{1: L} \hat{\mathbf{b}}_{1: L} \mid \hat{\tau}_{1: L \mid H_{1}}, H_{0}\right)}{p_{\mathbf{z}_{1: L} \mid H_{0}}\left(\mathbf{z}_{1: L} \mid \hat{\tau}_{1: L \mid H_{0}}, H_{0}\right)} \\
& =\frac{\prod_{r=1}^{L} \frac{1}{\hat{\tau}_{r \mid H_{1}}^{N}} \exp \left(-\frac{\left(\mathbf{z}_{r}-\hat{\mathbf{U}}_{r} \hat{\mathbf{b}}_{r}\right)^{H} \mathbf{M}^{-1}\left(\mathbf{z}_{r}-\hat{\mathbf{U}}_{r} \hat{\mathbf{b}}_{r}\right)}{\hat{\tau}_{r \mid H_{1}}}\right)}{\prod_{r=1}^{L} \frac{1}{\hat{\tau}_{r \mid H_{0}}^{N}} \exp \left(-\frac{\mathbf{z}_{r}^{H} \mathbf{M}^{-1} \mathbf{z}_{r}}{\hat{\tau}_{r \mid H_{0}}}\right)} .
\end{aligned}
$$

For notation convenience we name this detector DSM-GLRT for deterministic scatterer model GLRT.

The second approach models the scatterers' complex amplitude vector $\mathbf{b}_{r}$ as a complex Gaussian vector of unknown covariance matrix $\mathbf{b}_{r} \sim \mathcal{C N}\left(\mathbf{0}, \mathbf{R}_{\mathbf{b}_{r}}\right)$ so that the signal covariance matrix is $\mathbf{U}_{r} \mathbf{R}_{\mathbf{b}_{r}} \mathbf{U}_{r}^{H}$. This model has been adopted by McWhorter, et al. in [46] for their matched subspace detector for stochastic signals and also by Jin, et al. in [17], [47]. Under this 
second approach, the GLRT is expressed as

$$
\begin{aligned}
\Lambda_{\mathrm{GSM}-\mathrm{GLRT}}\left(\mathbf{z}_{1: L}\right) & =\frac{p_{\mathbf{z}_{1: L} \mid H_{1}}\left(\mathbf{z}_{1: L} \mid \hat{\mathbf{U}}_{1: L}, \hat{\mathbf{R}}_{\mathbf{b}_{1: L}}, \hat{\tau}_{1: L \mid H_{1}}, H_{1}\right)}{p_{\mathbf{z}_{1: L} \mid H_{0}}\left(\mathbf{z}_{1: L} \mid \hat{\tau}_{1: L \mid H_{0}}, H_{0}\right)} \\
& =\frac{\prod_{r=1}^{L} \frac{1}{\pi^{N} \operatorname{det}\left(\hat{\mathbf{B}}_{r}\right)} \exp \left(-\mathbf{z}_{r}^{H} \hat{\mathbf{B}}_{r}^{-1} \mathbf{z}_{r}\right)}{\prod_{r=1}^{L} \frac{1}{\tau_{r}^{N} \pi^{N} \operatorname{det}(\mathbf{M})} \exp \left(-\frac{\mathbf{z}_{r}^{H} \mathbf{M}^{-1} \mathbf{z}_{r}}{\hat{\tau}_{r \mid H_{0}}}\right)} .
\end{aligned}
$$

This second approach GLRT is named GSM-GLRT for Gaussian scatterer model GLRT. We notice that this GLRT derivation is more complicated due to the presence of $\operatorname{det}\left(\hat{\mathbf{B}}_{r}\right)=\operatorname{det}\left(\hat{\mathbf{U}}_{r} \hat{\mathbf{R}}_{\mathbf{b}_{r}} \hat{\mathbf{U}}_{r}^{H}+\hat{\tau}_{r} \mathbf{M}\right)$ and $\hat{\mathbf{B}}_{r}^{-1}=\left(\hat{\mathbf{U}}_{r} \hat{\mathbf{R}}_{\mathbf{b}_{r}} \hat{\mathbf{U}}_{r}^{H}+\hat{\tau}_{r} \mathbf{M}\right)^{-1}$.

\section{B. Signal Subspace Estimation with Superresolution Methods}

The signal subspace is determined by the columns of the matrices $\mathbf{E}_{r}$, and equivalently by the matrices of eigenvectors $\mathbf{U}_{r}$. We recall that indices $r$ vary between 1 and $L$; this is also true in the following. $\mathbf{E}_{r}$, the so-called steering matrices, contain all the Doppler components of the backscattered signal from the target and are then representative of the bidimensional target response. Their estimation are consequently an important step. The ML-estimation of the steering matrices $\mathbf{E}_{r}$ is not a straightforward problem. Indeed, no closed form exists and numerical methods must be used. We have studied an solution based on the expectation-maximization (EM) algorithm which enables to give an ML-estimate of the scatterers Doppler frequencies. The EM algorithm has been previously used in source separation [48-51] and radar imaging [52], [53]. We found that EM as a spectral estimator can give a better accuracy than superresolution methods, however, this technique exhibits a prohibitive computational complexity and convergence issues.

The previous point is precisely the reason why we resort to spectral analysis methods to estimate the signal subspace and more specifically to superresolution methods in order to give the most accurate estimation possible. These methods are also called subspace methods because their implementation is based on subspace eigendecomposition of the signal covariance matrix. These techniques need a white noise model and as a matter of fact, the measured signal vectors $\mathbf{z}_{r}=\mathbf{E}_{r} \mathbf{a}_{r}+\tau_{r} \mathbf{s}_{r}$ must be whitened so that $\tilde{\mathbf{z}}_{r}=\mathbf{M}^{-1 / 2} \mathbf{z}_{r}$ where $\mathbf{M}^{-1 / 2}$ is obtained through Cholesky factorization of $\mathbf{M}^{-1}$. Another solution is to use a frequency agile waveform that enables to decorrelate the speckle component. In each range cell, the measured signal covariance matrix then takes the form

$$
\mathbf{R}_{r}=\tilde{\mathbf{E}}_{r} \boldsymbol{\Sigma}_{r} \tilde{\mathbf{E}}_{r}^{H}+\tau_{r} \mathbf{I}
$$

where $\tilde{\mathbf{E}}_{r}=\mathbf{E}_{r}$ in the case of using frequency agility or is $\tilde{\mathbf{E}}_{r}=\mathbf{M}^{-1 / 2} \mathbf{E}_{r}$ after whitening.

The MUSIC algorithm [54] is based on the eigendecomposition of covariance matrix $\mathbf{R}_{r}$. With the signal model adopted in (3), $\tilde{\mathbf{E}}_{r} \boldsymbol{\Sigma}_{r} \tilde{\mathbf{E}}_{r}^{H}$ has $p_{r}$ positive eigenvalues and $N-p_{r}$ zero eigenvalues. Let the positive eigenvalues of $\tilde{\mathbf{E}}_{r} \Sigma_{r} \tilde{\mathbf{E}}_{r}^{H}$ be written

$$
\tilde{\lambda}_{1} \geq \tilde{\lambda}_{2} \geq \cdots \geq \tilde{\lambda}_{p_{r}} .
$$

The eigenvalues of $\mathbf{R}_{r}$ are

$$
\begin{aligned}
& \lambda_{k}=\tilde{\lambda}_{k}+\tau_{r}, \quad k=1, \ldots, p_{r} \\
& \lambda_{k}=\tau_{r}, \quad k=p_{r}+1, \ldots, N .
\end{aligned}
$$

The eigenvectors of $\mathbf{R}_{r}$ that correspond to the $p_{r}$ highest eigenvalues $\lambda_{1}, \ldots, \lambda_{p_{r}}$ span the same signal subspace as $\tilde{\mathbf{E}}_{r}$. Let $\tilde{\mathbf{U}}_{r}$ be the $N \times p_{r}$ matrix of the whitened signal subspace eigenvectors. The eigenvectors of $\mathbf{R}_{r}$ corresponding to the $N-p_{r}$ eigenvalues are orthogonal to the signal subspace and form the so-called noise subspace. Let $\tilde{\mathbf{U}}_{r}^{\perp}$ be the $N \times N-p_{r}$ noise subspace matrix.

The Doppler frequencies $f_{r, 1}, f_{r, 2}, \ldots, f_{r, p_{r}}$ of the $p_{r}$ components of signal $\mathbf{x}_{r}$ are then estimated by maximizing the MUSIC-pseudospectrum:

$$
S_{\text {MUSIC }}(f)=\frac{1}{\mathbf{e}(f)^{H} \tilde{\mathbf{U}}_{r}^{\perp} \tilde{\mathbf{U}}_{r}^{\perp H} \mathbf{e}(f)}
$$

where $\mathbf{e}(f)=\left(1, e^{j 2 \pi f}, \ldots, e^{j 2 \pi f(N-1)}\right)^{T}$. The Doppler frequencies are estimated as the locations of the $p_{r}$ highest peaks of the pseudospectrum.

From this point, we notice that we could directly inject the subspace eigenvectors matrix $\mathbf{U}_{r}$ into the GLRT. However, we found with simulations that it's necessary to constrain the structure of the signal subspace to $\mathbf{E}_{r}, r=1 \ldots L$, i.e., to implement the superresolution method so that it gives estimates of the Doppler frequencies, and after to compute the steering matrices $\mathbf{E}_{r}$. The subspace $\mathbf{U}_{r}$ extracted from the eigendecomposition is indeed sensitive to the estimation error of the correlation matrix $\mathbf{R}_{r}$ especially in small signal dimension cases, that is for small $N$ values.

Thus, we prefer the root-MUSIC or the ESPRIT algorithm [55] which gives directly estimates of the Doppler frequencies without maximizing the MUSIC pseudospectrum.

Finally, the signal subspace estimation is based on a specific model of the covariance matrix and needs the knowledge of the signal subspace dimension $p_{r}$, $r=1 \ldots L$. This knowledge is not obvious and must be estimated. The theory developed above tells us that $p_{r}$, $r=1 \ldots L$ corresponds to the number of eigenvalues higher than the smallest eigenvalue of the measured signal covariance matrix. However, the measured 
vector dimension $N$ being limited, the estimate of the covariance matrix is not precise and consequently, the model order selection is hard to determine. To avoid this limitation, several criteria coming from information theory have been proposed to estimate the signal subspace dimension or equivalently, the number of signal components. These criteria are the well-known Akaike information criterion (AIC) [56] or Rissanen's minimum description length (MDL) [57]. MDL is a consistent criterion. Moreover it is known that AIC tends to overestimate the signal subspace dimension, even in the presence of high signal-to-noise ratio, whereas MDL criterion tends to underestimate $p_{r}$ in the presence of low signal-to-noise ratio or a small number $N$ of measures. Let us mention that a strictly related problem has been considered by Gini and Bordini [58] who studied the behavior of such theoretical information criteria for model order selection in the presence of multiplicative noise. Several variations of AIC and MDL criteria have also been proposed even in the presence of colored noise but the signal subspace dimension remains a tricky issue.

\section{DSM-GLRT Expression}

After estimating the steering matrices $\mathbf{E}_{r}, r=$ $1 \ldots L$ and thus matrices $\mathbf{U}_{r}, r=1 \ldots L$, we are able to give the ML-estimates of the scatterers' complex amplitudes $\mathbf{b}_{r}, r=1 \ldots L$ and the texture component $\tau_{r}, r=1 \ldots L$. The details of the DSM-GLRT detector derivation are developed in Appendix A.

The GLRT is then given by

where

$$
\Lambda_{\mathrm{DSM}}(\mathbf{Z})=\frac{\prod_{r=1}^{L}\left(\mathbf{z}_{r}^{H} \mathbf{M}^{-1} \mathbf{z}_{r}\right)^{N}}{\prod_{r=1}^{L}\left(\mathbf{z}_{r}^{H}\left(\mathbf{M}^{-1}-\mathbf{Q}_{r}\right) \mathbf{z}_{r}\right)^{N}}
$$

$$
\mathbf{Q}_{r}=\mathbf{M}^{-1} \mathbf{U}_{r}\left(\mathbf{U}_{r}^{H} \mathbf{M}^{-1} \mathbf{U}_{r}\right)^{-1} \mathbf{U}_{r}^{H} \mathbf{M}^{-1}
$$

which is the orthogonal projector onto the signal subspace $\mathbf{U}_{r}$. In an equivalent way, the generalized $\log$-likelihood ratio is

$$
\ln \Lambda_{\mathrm{DSM}}(\mathbf{Z})=N \sum_{r=1}^{L} \ln \left(\frac{\mathbf{z}_{r}^{H} \mathbf{M}^{-1} \mathbf{z}_{r}}{\mathbf{z}_{r}^{H}\left(\mathbf{M}^{-1}-\mathbf{Q}_{r}\right) \mathbf{z}_{r}}\right) .
$$

It is interesting to note that the case where $r=1$ and the steering matrices are reduced to steering vectors gives a GLRT expression identical to that given in [27] in the case of a point target embedded in compound Gaussian clutter. Moreover, considering a range-only distributed target, i.e., only with one steering vector per range cell, we find an equivalent expression to the one derived in [20].

In order to draw a parallel with the following developments, we reexpress the DSM-GLRT with whitened data $\tilde{\mathbf{z}}_{r}=\mathbf{M}^{-1 / 2} \mathbf{z}_{r}, r=1, \ldots, L$ :

$$
\begin{aligned}
\ln \Lambda_{\mathrm{DSM}}(\mathbf{Z}) & =N \sum_{r=1}^{L} \ln \left(\frac{\tilde{\mathbf{z}}_{r}^{H} \tilde{\mathbf{z}}_{r}}{\tilde{\mathbf{z}}_{r}^{H}\left(\mathbf{I}-\tilde{\mathbf{Q}}_{r}\right) \tilde{\mathbf{z}}_{r}}\right) \\
& =N \sum_{r=1}^{L} \ln \left(1+\frac{\tilde{\mathbf{z}}_{r}^{H} \mathbf{P}_{\tilde{\mathbf{U}}_{r}} \tilde{\mathbf{z}}_{r}}{\tilde{\mathbf{z}}_{r}^{H} \mathbf{P}_{\tilde{\mathbf{U}}_{r}^{r}} \tilde{\mathbf{z}}_{r}}\right)
\end{aligned}
$$

where $\mathbf{P}_{\tilde{\mathbf{U}}_{r}}=\tilde{\mathbf{Q}}_{r}=\tilde{\mathbf{U}}_{r}\left(\tilde{\mathbf{U}}_{r}^{H} \tilde{\mathbf{U}}_{r}\right)^{-1} \tilde{\mathbf{U}}_{r}^{H}$ is the orthogonal projector onto the signal subspace and $\mathbf{P}_{\tilde{\mathbf{U}}_{r}^{\perp}}=\mathbf{I}-\mathbf{P}_{\tilde{\mathbf{U}}_{r}}$ is the orthogonal projector onto the clutter subspace with $\tilde{\mathbf{U}}_{r}=\mathbf{M}^{-1 / 2} \mathbf{U}_{r}$.

\section{GSM-GLRT Derivation}

The derivation of the GSM-GLRT is detailed in Appendix B. The log-likelihood ratio is then given by

$$
\begin{aligned}
& \ln \Lambda_{\mathrm{GSM}}(\mathbf{Z}) \\
&=-N L \ln N-\sum_{r=1}^{L} \ln \left(\left(N-p_{r}\right) \frac{\tilde{\mathbf{z}}_{r}^{H} \mathbf{P}_{\tilde{\mathbf{U}}_{r}} \tilde{\mathbf{z}}_{r}}{\tilde{\mathbf{z}}_{\tilde{\mathbf{U}}_{r}^{r}} \tilde{\mathbf{z}}_{r}}-\left(p_{r}-1\right)\right) \\
&+N \sum_{r=1}^{L} \ln \left(1+\frac{\tilde{\mathbf{z}}_{r}^{H} \mathbf{P}_{\tilde{\mathbf{U}}_{r}} \tilde{\mathbf{z}}_{r}}{\tilde{\mathbf{z}}_{r}^{H} \mathbf{P}_{\tilde{\mathbf{U}}_{r}^{+}} \tilde{\mathbf{z}}_{r}}\right) .
\end{aligned}
$$

We then notice that the GSM-GLRT is equivalent to the DSM-GLRT modified by the negative term. For $L=1$, the GSM-GLRT is a monotonic function of the ratio $\left(\tilde{\mathbf{z}}^{H} \mathbf{P}_{\tilde{\mathbf{U}}} \tilde{\mathbf{z}}\right) /\left(\tilde{\mathbf{z}}^{H} \mathbf{P}_{\tilde{\mathbf{U}} \perp} \tilde{\mathbf{z}}\right)$ and so is the DSM-GLRT; consequently the GSM-GLRT and DSM-GLRT are equivalent detectors. For $L>1$, Monte-Carlo trials described in Section V show that the performances of the two detectors are close.

REMARK 1 In the case of a Gaussian-compound disturbance, both the DSM and GSM GLRTs expressed in (21) and (22) are then a function of the ratio between the energy of the component of the whitened data which lies in the signal subspace, and the energy of the signal projected on noise subspace. DSM-GLRT simply integrates this ratio over all the range cells of the target.

REMARK 2 It is important to emphasize that the GLRT expression derived is CFAR with respect to the values of the texture component. This property is due to the deterministic model of $\tau_{r}, r=1 \ldots L$ and $\mathbf{b}_{r}, r=$ $1 \ldots L$ and their ML-estimation. The CFAR property is reached with the knowledge of the clutter covariance matrix M. This detector could be extended to a CFAR detector with respect to the clutter covariance matrix by resorting to a secondary data set as done by Conte, et al. in [15], [21]. 


\section{FALSE ALARM PROBABILITY AND THRESHOLD ASSESSMENT}

A detector displays the CFAR property when the detection threshold is independent of the clutter power. More generally, in the adaptive detection literature, the CFAR property refers to the clutter covariance matrix and more generally to all disturbance parameters that are unknown [16]. The derived detector is CFAR. Indeed, with the knowledge hypothesis of the covariance matrix $\mathbf{M}$ (estimated thanks to a secondary data set or being identity with an agile waveform), the GLRT is independent of the texture value. This is an important property which makes the detector adaptive. However, as we show in the following development, the detection threshold depends on the steering matrix and more precisely, of its rank i.e., the signal subspace dimension or the number of components.

We define the false alarm probability so that the log-likelihood ratio is higher than a threshold under $H_{0}$ :

$$
P_{\text {fa }}=\operatorname{Pr}\left\{\ln \Lambda\left(\mathbf{Z} \mid H_{0}\right)>\eta\right\} .
$$

We then distinguish the two cases for the DSM-GLRT.

1) For $L=1$, the $\log$-likelihood ratio is given by

$$
\Gamma=N \ln \left(1+\frac{\tilde{\mathbf{z}}^{H} \mathbf{P}_{\tilde{\mathbf{U}}} \tilde{\mathbf{z}}}{\mathbf{z}^{H} \mathbf{P}_{\tilde{\mathbf{U}}^{\perp}} \tilde{\mathbf{z}}}\right) \text {. }
$$

Under $H_{0}, \tilde{\mathbf{z}}=\tilde{\mathbf{c}}$. It is shown in [59] that, given a matrix $\mathbf{A}$, the quadratic form $\tilde{\mathbf{c}}^{H} \mathbf{A} \tilde{\mathbf{c}}$ is $\chi^{2}$ distributed with $2 p$ degrees of freedom, $p=\operatorname{rank}$ of $\mathbf{A}$, if and only if $\mathbf{A}$ is idempotent, i.e., $\mathbf{A}^{2}=\mathbf{A}$. With this property, the numerator of the ratio into brackets in (24) is $\chi^{2}$ distributed with $2 p$ degrees of freedom. Indeed, $\mathbf{P}_{\tilde{\mathbf{U}}}$ is an orthogonal projector onto the signal subspace of dimension $p$ and is thus idempotent. In the same way, $\mathbf{P}_{\tilde{\mathbf{U}}^{\perp}}$ is the orthogonal projector onto the clutter subspace and its rank is $N-p$. Consequently the denominator of the ratio in (24) is $\chi^{2}$ distributed with $2(N-p)$ degrees of freedom. Consequently:

$$
\begin{aligned}
\frac{\tilde{\mathbf{z}}^{H} \mathbf{P}_{\tilde{\mathbf{U}}} \tilde{\mathbf{z}}}{\mathbf{z}^{H} \mathbf{P}_{\tilde{\mathbf{U}}^{\perp}} \tilde{\mathbf{z}}} & \sim \frac{\chi^{2}(2 p)}{\chi^{2}(2 N-p)} \\
& \sim \frac{p}{N-p} F(2 p, 2(N-p))
\end{aligned}
$$

where $F(2 p, 2(N-p))$ is the well-known F-distribution. The probability density function (pdf) of the log-likelihood ratio under $H_{0}$ is then, thanks to the Jacobian transformation,

$$
p_{\Gamma}(\Gamma)=f_{1}(\Gamma)=\frac{p}{N-p} e^{\Gamma} F_{2 p, 2(N-p)}\left(\frac{N-p}{p}\left(e^{\Gamma}-1\right)\right) .
$$

2) For $L>1$, the log-likelihood ratio distribution is the convolution of previous distribution:

$$
p \Gamma(\Gamma)=f_{1}(\Gamma) * f_{2}(\Gamma) * \cdots f_{L}(\Gamma) .
$$

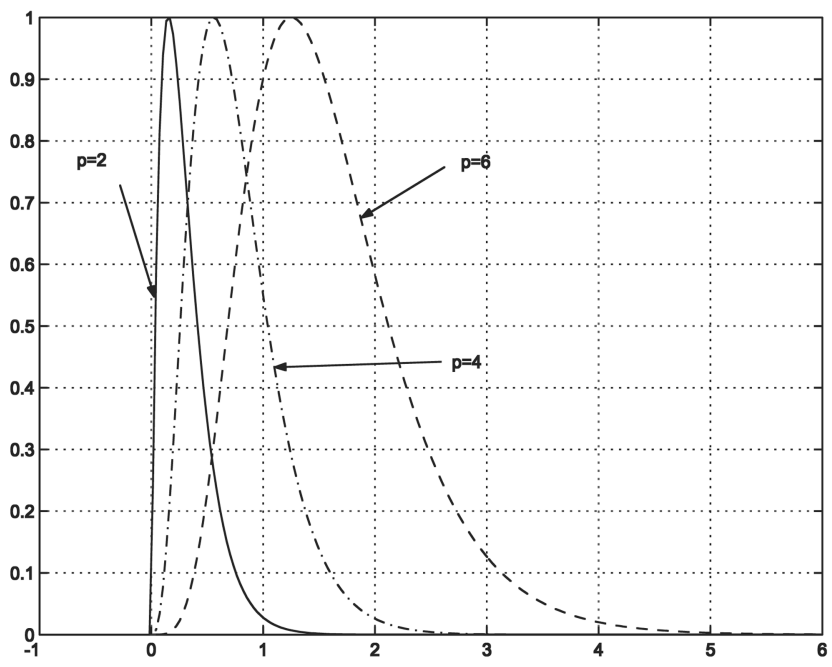

(a)

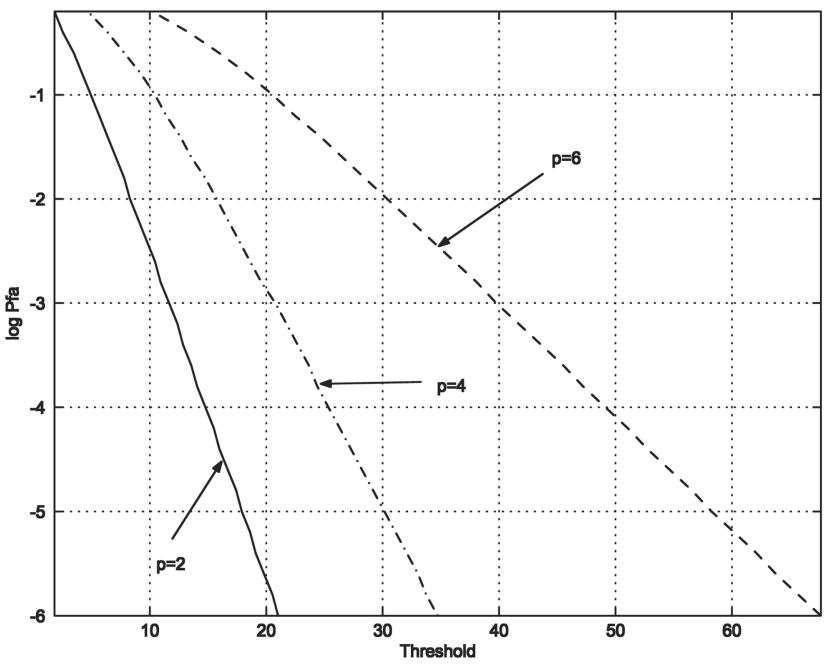

(b)

Fig. 1. (a) Normalized pdfs of log-likelihood ratio for $N=8$. (b) False alarm probability with respect to detection threshold for $L=1$ and $N=8$.

The GSM-GLRT false-alarm probability is more complicated to derive in a closed form. That is why we resort to Monte-Carlo simulations to compute it. Fig. 1 plots the normalized pdfs of the log-likelihood ratio, and false-alarm probability with respect to the threshold fixed for $N=8, L=1$ and different values of $p=0,2,4$, and 6 .

\section{SIMULATION RESULTS}

We present in this section the performances of both the DSM and GSM GLRT detectors on synthetic signals in different scenarios. The performances assessment is carried out resorting to computer simulations, i.e., we resort to Monte-Carlo simulations to estimate the detection probability and false alarm probabilities based on $100 / P_{\mathrm{d}}$ and $100 / P_{\mathrm{fa}}$ independent trials, which enables to obtain a good estimation 
TABLE I

Doppler Frequencies of Scatterers

\begin{tabular}{ccccc}
\hline \hline Cell \# & 1 & 2 & 3 & 4 \\
\hline Frequencies & $\{0.1\}$ & $\{0.1,0.2\}$ & $\{0.1,0.2,0.3\}$ & $\{0.1,0.2\}$ \\
\hline
\end{tabular}

accuracy. In order to limit the computational burden, the false alarm probability is chosen so that $P_{\mathrm{fa}}=10^{-4}$. In the different simulations, the DSM-GLRT is compared with the threshold computed by inverting the distribution given in (27) and the GSM-GLRT is compared with a threshold computed with Monte-Carlo trials. To save simulation time, we also consider small values of $N$ and $L$. Typically, the simulations consider $N=8$ pulses and $L=4$ range cells.

We consider a synthetic target which is distributed over $L=4$ range cells and in each range cell, the scatterers are located at different normalized Doppler frequencies as represented in Table I. For each scatterer, we fix unitary amplitudes. The scatterers amplitudes are considered constant during the simulation in most scenarios. We indeed study the case of random amplitudes in Section VE. Moreover, we attribute to each scatterer a random phase uniformly distributed in $[-\pi, \pi]$.

We assume in most tests that the corresponding steering matrices $\mathbf{E}_{r}, r=1, \ldots, L$ are known. This a priori knowledge is obviously not always straightforward and the signal subspace must be estimated. This aspect is studied in Section VG. The target total energy is then the sum of the energy in each range cell so that $\mathcal{E}=\sum_{r=1}^{L}\left\|\mathbf{E}_{r} \mathbf{a}_{r}\right\|^{2}$.

We assume here a clutter-dominating scenario. Increasing the radar resolution by a factor $L$, divides the clutter power in each range cell by the same factor $L$. The local value of texture $\tau_{r}, r=1 \ldots L$ is assumed to be gamma-distributed:

$$
p\left(\tau_{r}\right)=\frac{2 b^{\nu}}{\Gamma(\nu)} \tau_{r}^{2 \nu-1} \exp \left(-b^{2} \tau_{r}^{2}\right), \quad r=1 \ldots L
$$

where $b$ controls the mean of the distribution and $\nu$, the so-called shape parameter, controls the deviation with respect to the Gaussian distribution. The higher $\nu$ is, the more Gaussian the distribution. The statistics of a univariate clutter amplitude $c$ is then described by the well-known K-compound distribution:

$$
p_{c}(c)=\frac{2}{b \Gamma(\nu)}\left(\frac{c}{2 b}\right)^{\nu} K_{\nu-1}\left(\frac{c}{b}\right)
$$

where $K_{\nu}$ is the modified Bessel function of the second kind. This distribution models the sea statistics well [60]. Typical values of $\nu$ for sea clutter are greater than 0.4 [61]. In the following, the clutter covariance matrix is assumed to be identity so that

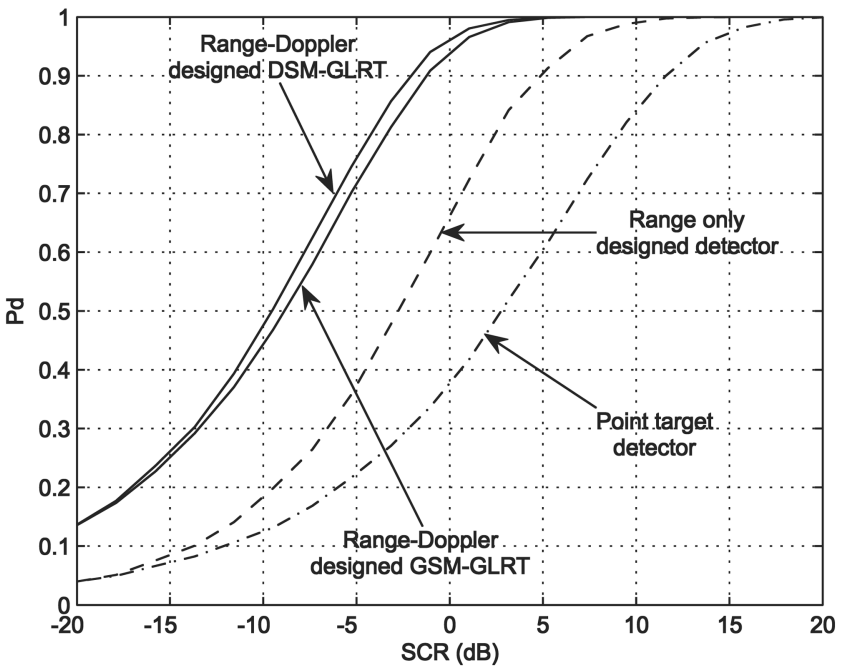

Fig. 2. Detection probabilities of DSM-GLRT and GSM-GLRT detectors designed for range- and Doppler-distributed targets (solid curves) with respect to point-target detector (dashdotted curve) and range-only distributed target detector (dashed curve) for $N=8$,

$L=4, \nu=0.5, P_{\mathrm{fa}}=10^{-4}$. Steering matrices and vectors are known.

$\mathbf{M}=\mathbf{I}$. The signal-to-clutter ratio (SCR) is

$$
\mathrm{SCR}=\frac{\sum_{r=1}^{L}\left(\mathbf{E}_{r} \mathbf{a}_{r}\right)^{H} \mathbf{M}^{-1}\left(\mathbf{E}_{r} \mathbf{a}_{r}\right)}{N \sigma^{2}}
$$

where $\sigma^{2}$ is the total energy of the clutter.

\section{A. Comparison with Other Detection Strategies}

In Fig. 2, the detection probabilities of our range-and-Doppler spread target detectors are plotted for $N=8, L=4$, and $\nu=0.5$. This shape parameter value corresponds to a spiky clutter. As the detection curves show, the performances of the GSM and DSM-GLRT detectors are nearly identical. In fact, in all the following simulations, the performances of the GSM and DSM detectors are very close, that is the reason why we only represent the DSM-GLRT detector performances. Next, in Fig. 2, the performances of the DSM and GSM are compared with the point-target-designed detector, i.e., the detector designed for $L=1$ which corresponds to a detector on a low or medium resolution range radar. We also assume that the steering matrix $\mathbf{E}_{1}=$ $\mathbf{e}_{1}=\left(1, e^{j 2 \pi f}, \ldots, e^{j 2 \pi f(N-1)}\right)$ reduces to a steering vector which frequency is fixed on $f=0.1$ which corresponds to the base of the target. This detector is then equivalent to [27]. In the point-target-designed detector case, the target is entirely located in one range cell and the clutter power is increased by a factor $L=4$ versus the range-and-Doppler spread target detector. We also plot the detection probability of the range-only distributed target detector, i.e., the detector designed for $L=4$ but in each range cell, the steering matrix is reduced to a steering vector so that $\mathbf{E}_{r}=\mathbf{e}_{r}=\left(1, e^{j 2 \pi f}, \ldots, e^{j 2 \pi f(N-1)}\right), r=1 \ldots L$ 


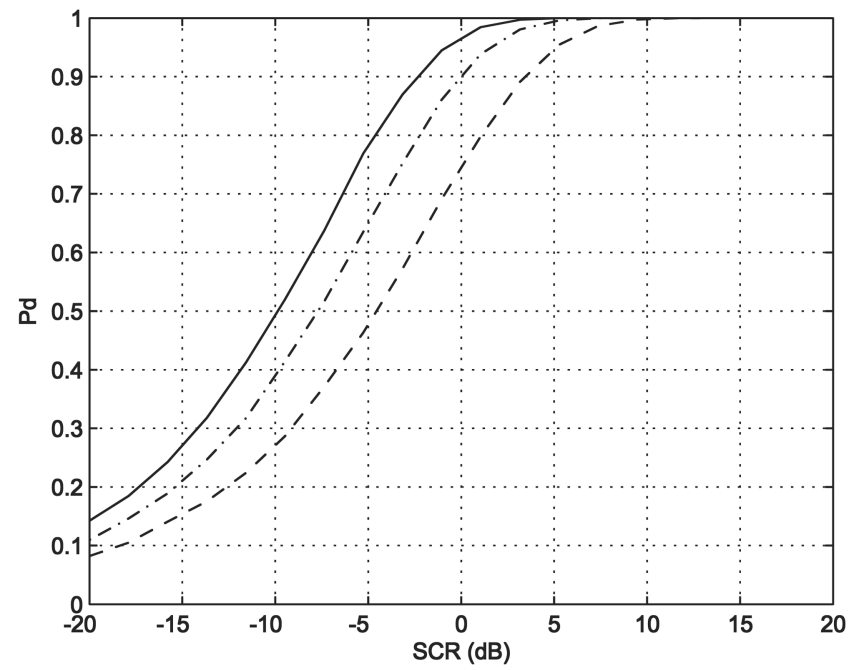

Fig. 3. Detection probability of DSM-GLRT detector for target 1 (solid curve), target 2 (dashdotted curve) and target 3 (dashed curve). $N=8, L=4, \nu=0.5, P_{\mathrm{fa}}=10^{-4}$. Steering matrices are known.

TABLE II

Doppler Frequencies and Amplitudes of the Scatterers

\begin{tabular}{c|ccccc}
\hline \hline & Cell & 1 & 2 & 3 & 4 \\
\hline target 1 & $f_{D}$ & $\{0.1,0.2\}$ & $\{0.1,0.2\}$ & $\{0.1,0.2\}$ & $\{0.1,0.2\}$ \\
& $a$ & $\{1,1\}$ & $\{1,1\}$ & $\{1,1\}$ & $\{1,1\}$ \\
\hline target 2 & $f_{D}$ & $\{0.1,0.2\}$ & $\{0.1,0.2\}$ & $\{0.1,0.2\}$ & $\{0.1,0.2\}$ \\
& $a$ & $\{2,2\}$ & $\{1,1\}$ & $\{1,1\}$ & $\{0,0\}$ \\
\hline target 3 & $f_{D}$ & $\{0.1,0.2\}$ & $\{0.1,0.2\}$ & $\{0.1,0.2\}$ & $\{0.1,0.2\}$ \\
& $a$ & $\{3,3\}$ & $\{1,1\}$ & $\{0,0\}$ & $\{0,0\}$ \\
\hline
\end{tabular}

where the steering vector frequency is $f=0.1$. This detector is equivalent to the one formulated in [20]. The figure shows that increasing radar resolution capabilities can produce a significant detection gain. Moreover, our detector (designed for target resolved on the Doppler axis) enables significant performance enhancement opposed to a detector designed for range-only distributed targets. Indeed, we observe a performance gain of $12 \mathrm{~dB}$ with respect to the point-target detector and approximatively $7 \mathrm{~dB}$ against the range-only distributed target detector, this gain being estimated for a detection probability of 0.5 .

\section{B. Influence of Mismatched Target Length}

Fig. 3 studies the consequences on detection probability of a mismatched length. To do so, we apply our DSM-GLRT detector on three different targets which Doppler frequencies and scatterers amplitudes are summarized in Table II. We witness a performance loss when the detector is not perfectly matched to the target. However, by comparing these results with Fig. 2, we can see that the performances are still higher than a range-only distributed detector or than a point-target detector.

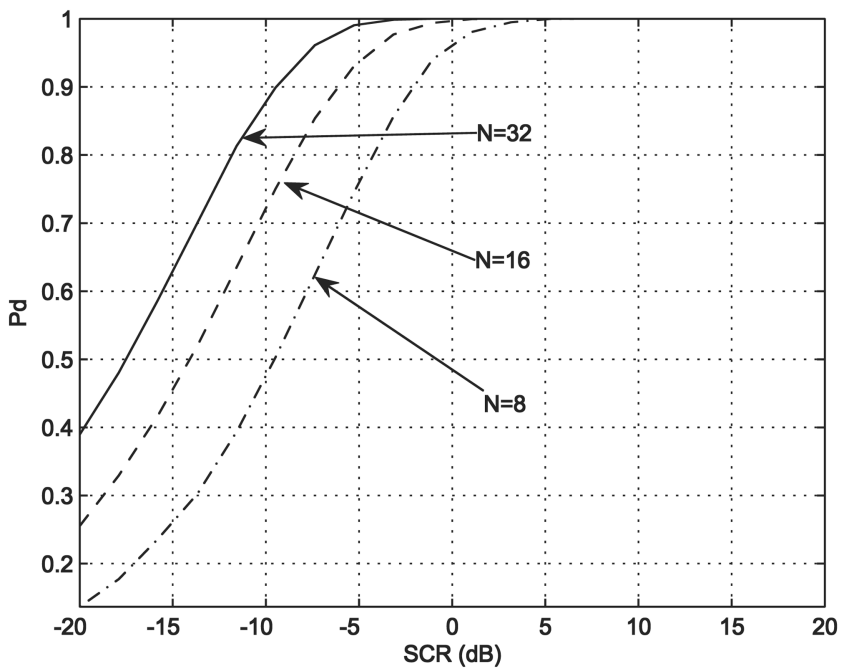

Fig. 4. Detection probability of DSM detector for different numbers of pulses used $N=8$ (dashdotted curve), $N=16$ (dashed curve) and $N=32$ (solid curve) for $L=4, \nu=0.5, P_{\mathrm{fa}}=10^{-4}$. Steering matrices are known.

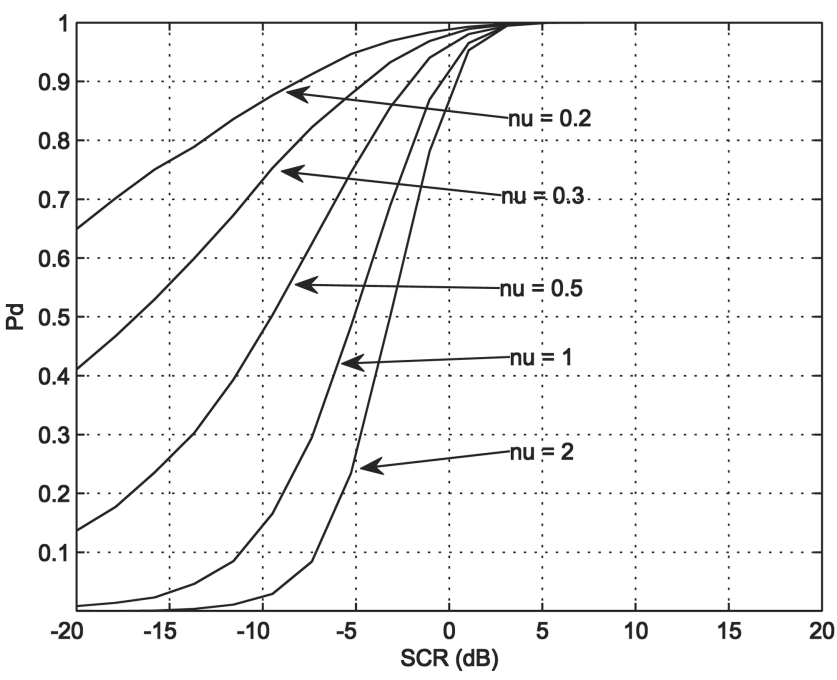

Fig. 5. Influence of $\nu$ on detection probability $P_{\mathrm{fa}}=10^{-4}, N=8$.

C. Influence of Number of Integrated Pulses

In Fig. 4 the detection probability is plotted for different values of $N=8,16$, and 32 . There is a large detection performance improvement when the number of pulses used is increased.

\section{Influence of Texture Shape Parameter $\nu$}

In Fig. 5, the detection probability is plotted for different shape parameters: $\nu=1, \nu=0.5, \nu=$ 0.3 , and $\nu=0.2$. We can note that the detection probability increases when the clutter becomes more spiky, especially for low SCR. This result about the influence of $\nu$ is also observed in [20] and [21] for range-distributed detectors and in [62] for point target detector. 


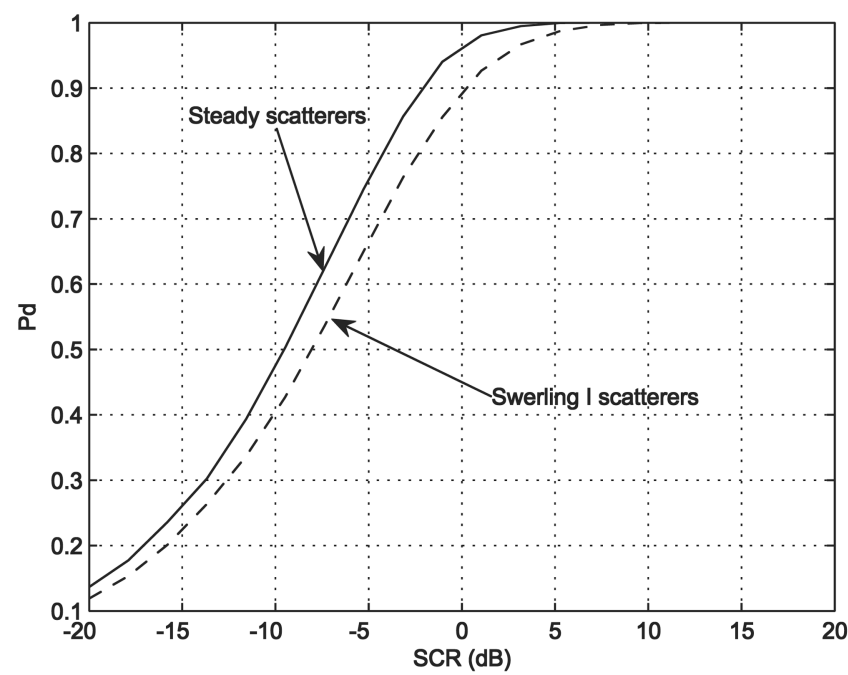

Fig. 6. Comparison of DSM-GLRT on steady scatterers (solid curve) and Swerling I scatterers (dashed curve). $P_{\mathrm{fa}}=10^{-4}, N=8$, $\nu=0.5$.

\section{E. Influence of Target Amplitudes Fluctuations}

We assumed in previous Monte-Carlo simulations that the scattering points' amplitudes were unitary and constant over the simulation time. In Fig. 6, a fluctuation is introduced on the amplitudes considering that they follow a zero-mean Gaussian distribution with unit variance. The amplitudes vary between each simulation but are constant over the $N$ pulses. This model corresponds to Swerling I [25] for a single scatterer. The detection probability is slightly lower but stays very close to the case of constant amplitudes. The detection loss is approximately of $2 \mathrm{~dB}$ for $P_{\mathrm{d}}=0.5$.

\section{F. Influence of the Texture Correlation}

In the problem statement (Section II), we assumed that the different range cells were independent. In some scenarios, this assumption may no longer be valid and it is interesting to evaluate the detection loss when a spatial correlation is introduced. Fig. 7 plots the detection probability versus the ratio between the correlation length and the target length. The target now considered lies in $L=16$ range cells. The Doppler frequencies of the scattering points in range cells $\{1,2,3,4\}$ are equal to $\{0.1\}$, in range cells $\{5,6,7,8\}$ they are equal to $\{0.1,0.2\}$, in range cells $\{9,10,11,12\}$ they are equal to $\{0.1,0.2,0.3\}$ and in range cells $\{13,14,15,16\}$ they are are equal to $\{0.1,0.2\}$. The detection probability is plotted for $\mathrm{SCR}=-10 \mathrm{~dB}$ and, as we can see, it decreases when the correlation length increases. The spatial correlation of the gamma texture component is simulated via a memoryless nonlinear transform (MNLT) as described in [63]. The correlation length corresponds to the value for which the autocorrelation function is equal to $1 / e$. Gerlach [20] proposed to pass the different

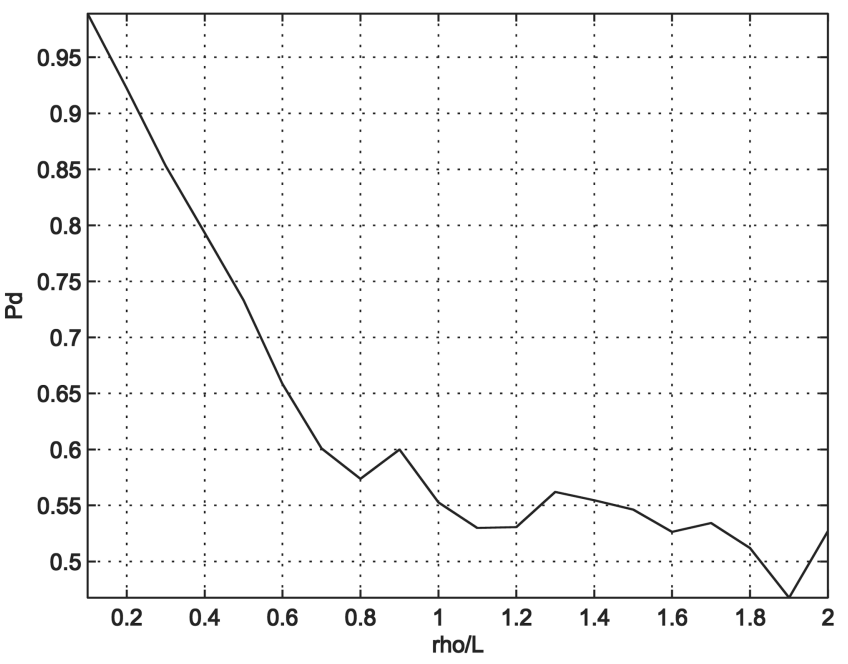

Fig. 7. Influence of correlation length of texture component. $\mathrm{SCR}=-10 \mathrm{~dB}, P_{\mathrm{fa}}=10^{-4}, N=8, \nu=0.5, L=16$.

pulses through a whitening filter before the detection stage. The spatial whitening takes then the form of a whitening matrix derived from the Cholesky decomposition of the covariance matrix of the input samples in range on a single pulse. The spatial correlation function could also be computed thanks to a secondary data set were it available.

\section{G. Influence of Signal Subspace Estimation}

In previous simulations, steering matrices or steering vectors were assumed to be known. In a realistic scenario, this a priori knowledge is obviously not always straightforward and the signal subspace must be estimated. We have proposed in Section IIIB to use superresolution methods to estimate the Doppler frequencies of the signal components. These methods need the knowledge of the signal subspace dimension, i.e., the number of signal components. To do so, we use Rissanen's MDL [57] criterion which solve the surestimation problem encountered with AIC [56] (MDL however tends to underestimate the number of components for a small signal length). We use Least Square ESPRIT [55] and root-MUSIC which enables to give an estimate of the Doppler frequencies without having to search over the maxima of a function as is the case when using the MUSIC algorithm [54]. In Fig. 8, the detection probability is plotted using on the one hand the ESPRIT algorithm for the estimation, and on the other hand the root-MUSIC algorithm. In order to save simulation time, the considered target is contained in one range cell and is composed of three unitary scattering points of respective normalized Doppler frequencies $\{0.1,0.2,0.3\}$. The computed threshold corresponds to a wanted $P_{\mathrm{fa}}=10^{-2}$. The data length is $N=64$ samples in order to limit the frequency estimation error. The correlation matrix is estimated thanks to a forward-backward averaging or a spatial smoothing technique [64]. 


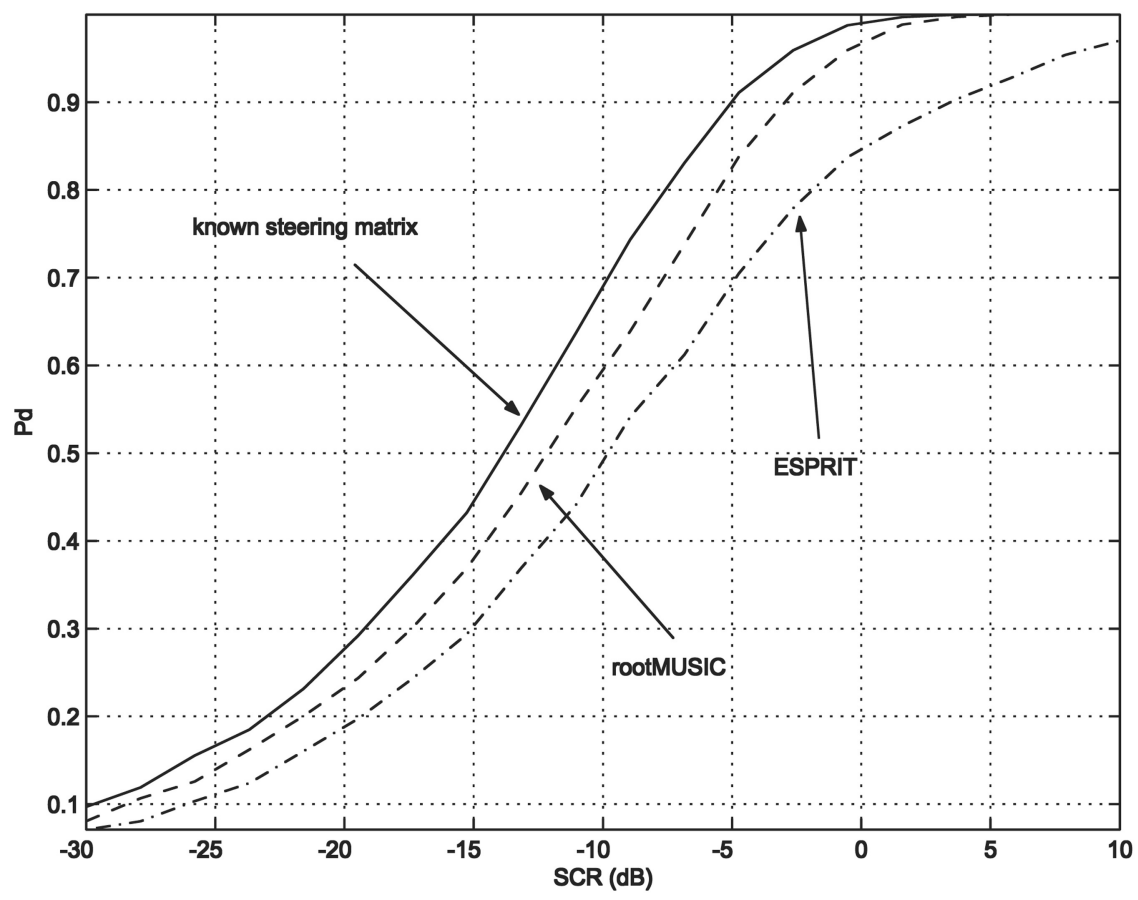

Fig. 8. Comparison of DSM-GLRT on target composed of 3 frequencies in one range cell in case where frequencies are known (solid curve), ESPRIT-estimated (dashdotted curve) and root-MUSIC-estimated (dashed curve). $P_{\mathrm{fa}}=10^{-2}, N=64, \nu=0.5, L=1$.

Forward-backward averaging exploits the fact that a sinusoid evolves in one spatial direction in the same manner as the conjugate sinusoid evolves in the opposite spatial direction, while conjugating and reversing the clutter/noise contribution effectively yields an independent realization, thereby doubling the amount of averaging that occurs [65]. The correlation matrix estimate of the data sequence $\mathbf{z}_{r}=\left(z_{r}(0), z(1), \ldots, z(N-1)\right)^{t}$ is then computed by forming subsequences $\mathbf{z}_{r, k}, k=1 \ldots K$ of lengths $N / K$. The estimate is then given by

$$
\hat{\mathbf{R}}_{\mathbf{z}_{r}}=\frac{1}{2 K}\left(\sum_{k=1}^{K} \mathbf{Z}_{r, k}+\mathbf{J} \mathbf{Z}_{r, k} \mathbf{J}\right), \quad r=1 \ldots L
$$

where $\mathbf{Z}_{r, k}=\mathbf{z}_{r, k} \mathbf{z}_{r, k}^{H}$ and $\mathbf{J}$, the exchange matrix that makes $\mathbf{J} \mathbf{z}_{r, k}$ the reverse version of $\mathbf{z}_{r, k}$, is defined as

$$
\mathbf{J}=\left(\begin{array}{cccc}
0 & \cdots & 0 & 1 \\
0 & \cdots & 1 & 0 \\
\vdots & / & 0 & \vdots \\
1 & 0 & \cdots & 0
\end{array}\right) .
$$

In the simulations, we set $K=2$. It is indeed shown in [66] that the best compromise between the resolution loss and the decorrelation of different contributors is reached for $2 \leq K \leq 3$.

In order to take into account the frequency estimation error, we define the detection probability as the probability that the GLRT be higher than the threshold defined earlier and that the frequency estimation error defined in (33) and (34) be lower than the arbitrarily fixed value $1 / N$, corresponding to the periodogram frequency resolution. Fig. 8 shows that estimating the steering matrix yields a detection loss that depends on the frequency estimation accuracy.

$$
\text { If } \hat{p}_{r} \leq p_{r} \text { : }
$$

$$
\text { err }=\frac{1}{L} \sum_{r=1}^{L} \frac{1}{\hat{p}_{r}} \sum_{k=1}^{\hat{p}_{r}} \min _{i}\left|\hat{f}_{k}-f_{i}\right|
$$

otherwise:

$$
\text { err }=\frac{1}{L} \sum_{r=1}^{L} \frac{1}{p_{r}} \sum_{k=1}^{p_{r}} \min _{i}\left|\hat{f}_{i}-f_{k}\right| .
$$

Actually, the estimation error on frequency affects also the false alarm probability. Indeed, frequency estimation errors or errors on the number of signal components entail including clutter components in the steering matrix. As a consequence, the false alarm probability increases. The influence on mismatched steering vectors is tackled in [67], [16] and more recently by De Maio in [68], the authors compute the false alarm probability and detection probability as a function of the angle between real and estimated steering vectors. In Fig. 9, we plot the logarithm of the root mean square error (RMSE) on frequency estimation and of the Monte-Carlo computed $P_{\mathrm{fa}}$. By looking at Fig. 9, we understand that the false alarm probability is linked to the error on frequency estimation. ESPRIT RMSE is higher than root-MUSIC RMSE for SCRs above $0 \mathrm{~dB}$. The opposite behavior is usually encountered, see for example [69], but 


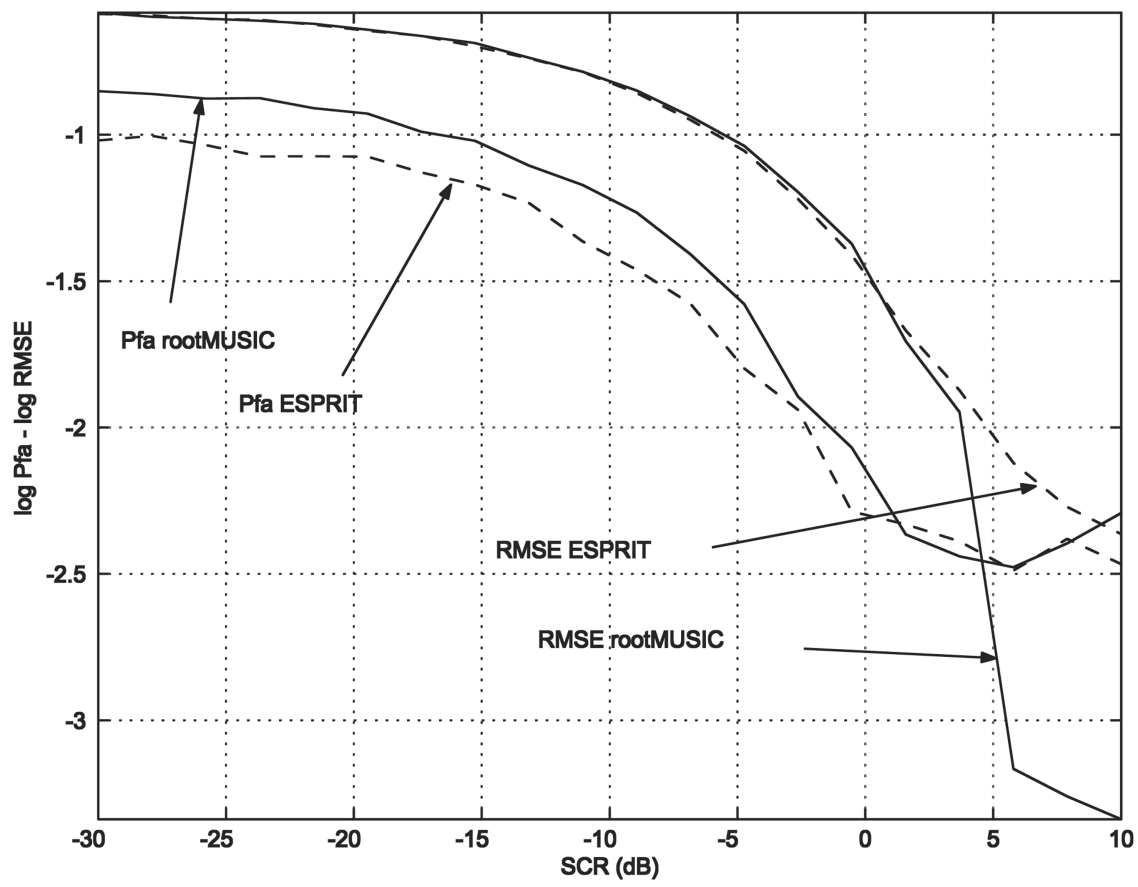

Fig. 9. Logarithm of root mean square error on frequency estimation and of computed $P_{\mathrm{fa}}$ (dashed curve). $P_{\mathrm{fa}}=10^{-2}, N=64, \nu=0.5$, $L=1$. Signal subspace dimension is estimated thanks to MDL criterion.

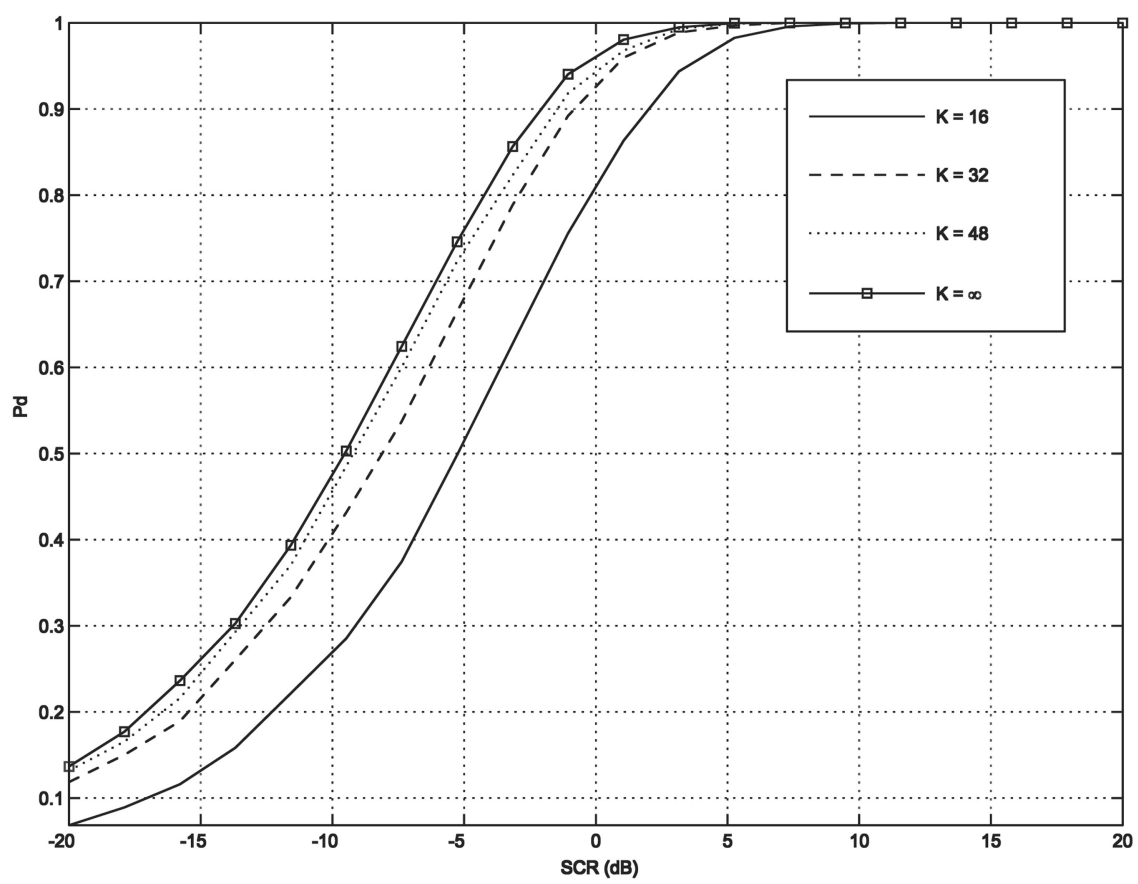

Fig. 10. Detection probability with estimation of covariance matrix from $K=16, K=32, K=48$ secondary clutter data vectors. $N=8$, $L=4, \nu=0.5, P_{\mathrm{fa}}=10^{-4}$. Steering matrices are known.

the test we made includes estimation of the signal subspace thanks to Rissanen's criterion. In fact, when the signal subspace dimension is estimated thanks to the Rissanen's MDL, we witness that for SCRs comprised between $0 \mathrm{~dB}$ and approximately $20 \mathrm{~dB}$, the ESPRIT RMSE is higher than the root-MUSIC RMSE ; and when the target dominates the clutter, i.e., for SCRs higher than $20 \mathrm{~dB}$, normal behavior is observed, i.e., the ESPRIT RMSE is slightly lower than the root-MUSIC RMSE.

\section{H. Influence of Additive Thermal Noise and Covariance Matrix Estimation}

In all previous simulations, we assumed that the clutter covariance matrix is known. In a realistic 


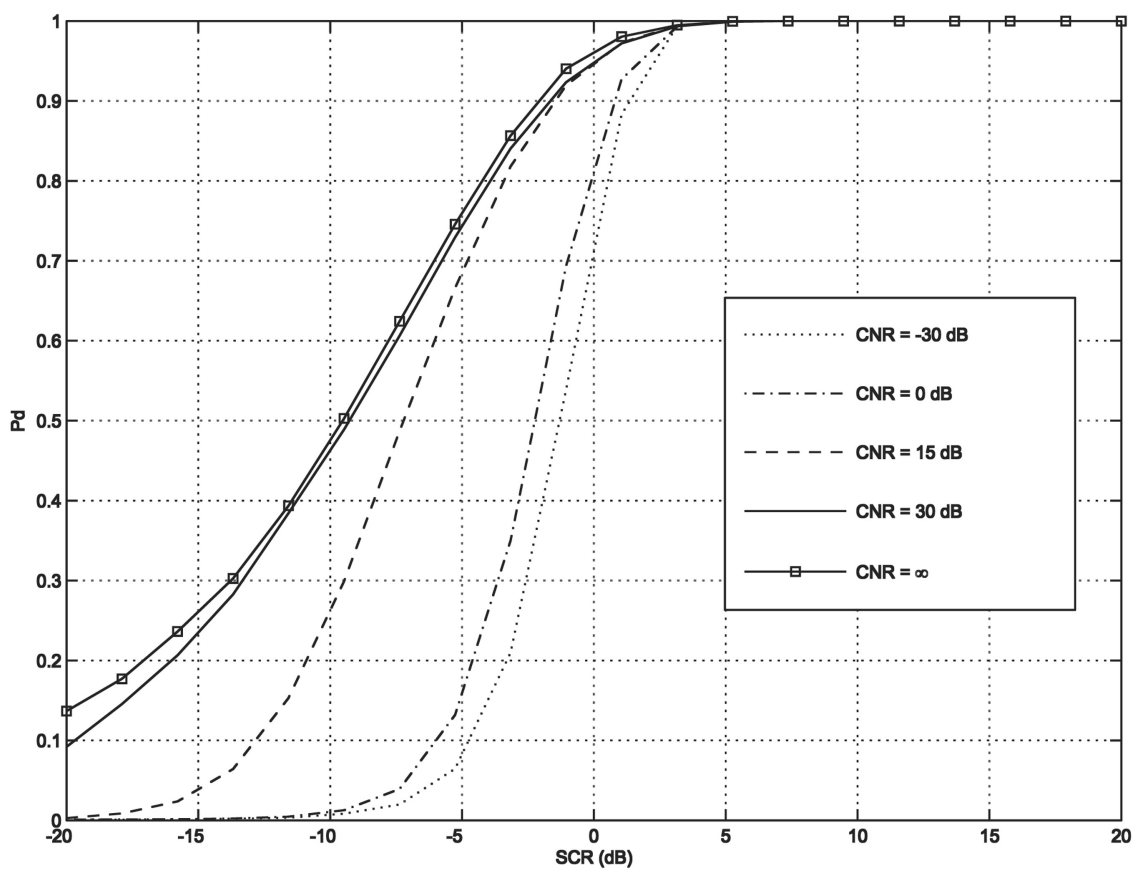

Fig. 11. Influence of clutter to thermal noise ratio (CNR). Covariance matrix estimated from $K=32$ clutter+noise secondary data vectors. $N=8, L=4, \nu=0.5, P_{\mathrm{fa}}=10^{-4}$. Steering matrices are known.

scenario, it must be estimated from a secondary data set. In this section, we present the performances of the derived detector with the estimation of the covariance matrix from secondary data only composed of clutter returns, denoted $\mathbf{z}_{r}=\sqrt{\tau_{r}} \mathbf{s}_{r}, r=L+1 \ldots L+H$. The covariance matrix is supposed to be the same in the different range cells and the estimate is then computed from the normalized data so that

$$
\hat{\mathbf{M}}=\frac{1}{H} \sum_{k=L+1}^{L+H} \frac{\mathbf{z}_{r} \mathbf{z}_{r}^{H}}{\frac{1}{N} \mathbf{z}_{r}^{H} \mathbf{z}_{r} .}
$$

This estimate is also used by Conte, De Maio, and Ricci in [21]. The estimation of the covariance matrix leads to a different threshold assessment. In fact, no closed-form exists and we resort to Monte-Carlo trials to estimate the new threshold. Fig. 10 then shows the detection curves when secondary data is composed of $K=16, K=32$ or $K=48$ clutter vectors and these curves are compared to the case of a known covariance matrix, which is written $K=\infty$. As the figure shows, the detection probability decreases when the covariance matrix is estimated. However, with a sufficient number of secondary data vectors, the performances corresponding to an estimated covariance matrix become very close the performances corresponding to a known covariance matrix.

Next, in the problem statement, we addressed the problem of detecting targets in a clutter-dominant scenario, that is, we neglected the influence of thermal noise. This assumption can be made if we consider low-range detections since the influence of thermal noise increases with range. We thus plot in Fig. 11 the detection performances of the proposed detector when thermal noise is added to the disturbance vectors. In that case, the covariance matrix is estimated via (35) from $K=32$ secondary data vectors composed of clutter and thermal noise. We see that the detection probability decreases when the clutter-to-noise ratio decreases. Actually, this phenomenon may be explained considering the mixture of clutter and thermal noise. Indeed, the more powerful the thermal noise, the more the distribution of the mixture tends to Gaussian-shape statistics. Otherwise stated, this situation makes the detector behave in the same way as an increase of the shape parameter $\nu$ would do.

\section{CONCLUSION}

We have proposed in this paper an adaptive detector for range- and Doppler-spread targets in non-Gaussian disturbance. The clutter is modeled as a SIRP with known or estimated speckle covariance matrices. The target steering matrices are also assumed to be known or estimated with spectral estimators such as the root-MUSIC or ESPRIT superresolution methods. We consider that the local values of the texture component are deterministic unknown parameters and are ML-estimated so that the GLRT can be derived. Two different hypotheses have been made concerning the complex amplitudes of the target scatterers: either modeled as deterministic unknowns or as Gaussien parameters. It has been analytically shown that both hypotheses lead to the same results as for a single range cell designed detector and Monte-Carlo trials show that results 
are very close for distributed target designed detectors. We have shown that our detector, which is especially designed for range- and Doppler-distributed targets enables a performance enhancement over low-resolution detection or range-only-target-designed detectors and that our detector has the CFAR property. Our interest is now focused on investigating the possibility of using our detector to estimate the target length by maximizing the likelihood with respect to a sliding window of variable length and to extend the binary-hypotheses test to a multiple-hypotheses test, which should enable to differentiate different kinds of targets.

\section{APPENDIX A. DERIVATION OF THE DSM-GLRT}

The ML-estimate of $\mathbf{b}_{1 \ldots L}$ under hypothesis $H_{1}$ is given by

$$
\begin{aligned}
\hat{\mathbf{b}}_{r} & =\underset{\mathbf{b}_{r}}{\arg \max } p_{\mathbf{z}_{r} \mid \mathbf{b}_{r}, \mathbf{U}_{r}, \tau_{r}, H_{1}}\left(\mathbf{z}_{r} \mid \mathbf{b}_{r}, \mathbf{U}_{r}, \tau_{r}, H_{1}\right) \\
& =\underset{\mathbf{b}_{r}}{\arg \max } p_{\mathbf{z}_{r}-\mathbf{U}_{r} \mathbf{b}_{r} \mid \tau_{r}, H_{0}}\left(\mathbf{z}_{r}-\mathbf{U}_{r} \mathbf{b}_{r} \mid \tau_{r}, H_{0}\right) .
\end{aligned}
$$

The result is thus simply given by computing and cancelling the likelihood derivative with respect to $\mathbf{b}_{r}$. This is equivalent to computing the derivative in each range cell so that

$$
\begin{gathered}
\frac{\partial}{\partial \mathbf{b}_{r}}\left[\frac{\exp \left(-\left(\mathbf{z}_{r}-\mathbf{U}_{r} \mathbf{b}_{r}\right)^{H} \mathbf{M}^{-1}\left(\mathbf{z}_{r}-\mathbf{U}_{r} \mathbf{b}_{r}\right) / \tau_{r}\right)}{\left(\pi \tau_{r}\right)^{N} \operatorname{det}(\mathbf{M})}\right]=0 \\
\Leftrightarrow \hat{\mathbf{b}}_{r}=\left(\mathbf{U}_{r}^{H} \mathbf{M}^{-1} \mathbf{U}_{r}\right)^{-1} \mathbf{U}_{r}^{H} \mathbf{M}^{-1} \mathbf{z}_{r}, \\
r=1 \ldots L .
\end{gathered}
$$

The same approach is adopted to find the ML-estimate of the texture $\tau_{1 \ldots L}$ under $H_{0}$ and $H_{1}$. It is straightforward to show that the estimates are, respectively, given by

$$
\begin{aligned}
\hat{\tau}_{r \mid H_{0}} & =\underset{\tau_{r}}{\arg \max } p_{\mathbf{z}_{r} \mid \tau_{r}, H_{0}}\left(\mathbf{z}_{r} \mid \tau_{r}, H_{0}\right) \\
& =\frac{\mathbf{z}_{r}^{H} \mathbf{M}^{-1} \mathbf{z}_{r}}{N}
\end{aligned}
$$

and, with (37)

$$
\begin{aligned}
\hat{\tau}_{r \mid H_{1}} & =\underset{\tau_{r}}{\arg \max } p_{\mathbf{z}_{r} \mid \tau_{r}, H_{1}}\left(\mathbf{z}_{r} \mid \tau_{r}, H_{1}\right) \\
& =\frac{\mathbf{z}_{r}^{H}\left(\mathbf{M}^{-1}-\mathbf{Q}_{r}\right) \mathbf{z}_{r}}{N}
\end{aligned}
$$

where

$$
\mathbf{Q}_{r}=\mathbf{M}^{-1} \mathbf{U}_{r}\left(\mathbf{U}_{r}^{H} \mathbf{M}^{-1} \mathbf{U}_{r}\right)^{-1} \mathbf{U}_{r}^{H} \mathbf{M}^{-1}
$$

which is the orthogonal projector onto the signal subspace $\mathbf{U}_{r}$. By injecting the ML-estimate of (37), (38), and (39) in (12), the GLRT is reexpressed as

$$
\Lambda_{\mathrm{DSM}}(\mathbf{Z})=\frac{\prod_{r=1}^{L}\left(\mathbf{z}_{r}^{H} \mathbf{M}^{-1} \mathbf{z}_{r}\right)^{N}}{\prod_{r=1}^{L}\left(\mathbf{z}_{r}^{H}\left(\mathbf{M}^{-1}-\mathbf{Q}_{r}\right) \mathbf{z}_{r}\right)^{N}} .
$$

\section{APPENDIX B. DERIVATION OF THE GSM-GLRT}

Let us consider the whitened data $\tilde{\mathbf{z}}_{r}=\mathbf{M}^{-1 / 2} \mathbf{z}_{r}$, $r=1 \ldots L$ and the whitened signal covariance matrix $\tilde{\mathbf{R}}_{\mathbf{x}_{r}}=\mathbf{M}^{-1 / 2} \mathbf{U}_{r} \mathbf{R}_{\mathbf{b}_{r}} \mathbf{U}_{r}^{H} \mathbf{M}^{-1 / 2}=\tilde{\mathbf{U}}_{r} \mathbf{R}_{\mathbf{b}_{r}} \tilde{\mathbf{U}}_{r}^{H}$. With this strategy, the likelihood under $H_{1}$ is

$$
f_{1}(\mathbf{Z})=\prod_{r=1}^{L} \frac{\exp \left(-\tilde{\mathbf{z}}_{r}^{H}\left(\tilde{\mathbf{U}}_{r} \mathbf{R}_{\mathbf{b}_{r}} \tilde{\mathbf{U}}_{r}^{H}+\tau_{r} \mathbf{I}\right)^{-1} \tilde{\mathbf{z}}_{r}\right)}{\pi^{N} \operatorname{det}\left(\tilde{\mathbf{U}}_{r} \mathbf{R}_{\mathbf{b}_{r}} \tilde{\mathbf{U}}_{r}^{H}+\tau_{r} \mathbf{I}\right)} .
$$

In order to compute $\operatorname{det}\left(\tilde{\mathbf{U}}_{r} \mathbf{R}_{\mathbf{b}_{r}} \tilde{\mathbf{U}}_{r}^{H}+\tau_{r} \mathbf{I}\right)$ and $\left(\tilde{\mathbf{U}}_{r} \mathbf{R}_{\mathbf{b}_{r}} \tilde{\mathbf{U}}_{r}^{H}+\tau_{r} \mathbf{I}\right)^{-1}$ and to derive the GSM-GLRT, we follow the same approach as McWhorter [46] and Jin [17], [47]. For this purpose, we present the signal covariance matrix in a different form. Let us write

$$
\tilde{\mathbf{R}}_{\mathbf{b}_{r}}=\left(\tilde{\mathbf{U}}_{r}^{H} \tilde{\mathbf{U}}_{r}\right)^{1 / 2} \mathbf{R}_{\mathbf{b}_{r}}\left(\tilde{\mathbf{U}}_{r}^{H} \tilde{\mathbf{U}}_{r}\right)^{1 / 2}=\mathbf{G}_{r} \mathbf{D}_{r} \mathbf{G}_{r}^{H}
$$

where $\mathbf{G}_{r}$ is the unitary matrix of eigenvectors of this new form and $\mathbf{D}_{r}=\operatorname{diag}\left[d_{r, 1}, d_{r, 2}, \ldots, d_{r, p_{r}}\right]$ its eigenvalues. Then we define

$$
\tilde{\mathbf{G}}_{r}=\tilde{\mathbf{U}}_{r}\left(\tilde{\mathbf{U}}_{r}^{H} \tilde{\mathbf{U}}_{r}\right)^{-1 / 2} \mathbf{G}_{r}=\left[\mathbf{g}_{r, 1}, \ldots, \mathbf{g}_{r, p_{r}}\right] .
$$

It is straightforward to see that the columns of $\tilde{\mathbf{G}}_{r}$ are a set of orthonormal vectors. Let $\tilde{\mathbf{G}}_{r}^{\perp}$ denote the null space of $\tilde{\mathbf{G}}_{r}$ of dimension $N \times\left(N-p_{r}\right)$. Thus we have $\tilde{\mathbf{G}}_{r}^{\perp H}\left[\tilde{\mathbf{G}}_{r} \tilde{\mathbf{G}}_{r}^{\perp}\right]=\left[\mathbf{0}_{\left(N-p_{r}\right) \times p_{r}} \mathbf{I}_{\left(N-p_{r}\right) \times\left(N-p_{r}\right)}\right]$. The covariance matrix $\tilde{\mathbf{U}}_{r} \mathbf{R}_{\mathbf{b}_{r}} \tilde{\mathbf{U}}_{r}^{H}+\tau_{r} \mathbf{I}$ can then be written

$$
\tilde{\mathbf{U}}_{r} \mathbf{R}_{\mathbf{b}_{r}} \tilde{\mathbf{U}}_{r}^{H}+\tau_{r} \mathbf{I}=\left[\tilde{\mathbf{G}}_{r} \tilde{\mathbf{G}}_{r}^{\perp}\right]\left[\begin{array}{cc}
\mathbf{D}_{r}+\tau_{r} \mathbf{I}_{p_{r}} & \mathbf{0} \\
\mathbf{0} & \tau_{r} \mathbf{I}_{N-p_{r}}
\end{array}\right]\left[\tilde{\mathbf{G}}_{r} \tilde{\mathbf{G}}_{r}^{\perp}\right]^{H} .
$$

It follows that

$$
\begin{aligned}
\left(\tilde{\mathbf{U}}_{r} \mathbf{R}_{\mathbf{b}_{r}} \tilde{\mathbf{U}}_{r}^{H}+\tau_{r} \mathbf{I}\right)^{-1} & \\
= & {\left[\tilde{\mathbf{G}}_{r} \tilde{\mathbf{G}}_{r}^{\perp}\right]\left[\begin{array}{cc}
\left(\mathbf{D}_{r}+\tau_{r} \mathbf{I}_{p_{r}}\right)^{-1} & \mathbf{0} \\
\mathbf{0} & \frac{1}{\tau_{r}} \mathbf{I}_{N-p_{r}}
\end{array}\right]\left[\tilde{\mathbf{G}}_{r} \tilde{\mathbf{G}}_{r}^{\perp}\right]^{H} } \\
& =\sum_{k=1}^{p_{r}} \frac{1}{d_{r, k}+\tau_{r}} \mathbf{P}_{\tilde{\mathbf{g}}_{r, k}}+\frac{1}{\tau_{r}} \mathbf{P}_{\tilde{\mathbf{G}}_{r}^{\perp}}
\end{aligned}
$$

where

$$
\begin{gathered}
\mathbf{P}_{\tilde{\mathbf{g}}_{r, k}}=\tilde{\mathbf{g}}_{r, k} \tilde{\mathbf{g}}_{r, k}^{H} \\
\mathbf{P}_{\tilde{\mathbf{G}}_{r}^{\perp}}=\tilde{\mathbf{G}}_{r}^{\perp} \tilde{\mathbf{G}}_{r}^{\perp H}=\mathbf{I}-\mathbf{P}_{\tilde{\mathbf{G}}_{r}}=\mathbf{I}-\tilde{\mathbf{G}}_{r} \tilde{\mathbf{G}}_{r}^{H}
\end{gathered}
$$

and it can be verified that we also have $\mathbf{P}_{\tilde{\mathbf{G}}_{r}}=\mathbf{P}_{\tilde{\mathbf{U}}_{r}}$. In the same way, $\operatorname{det}\left(\tilde{\mathbf{U}}_{r} \mathbf{R}_{\mathbf{b}_{r}} \tilde{\mathbf{U}}_{r}^{H}+\tau_{r} \mathbf{I}\right)^{-1}$ is given by

$$
\operatorname{det}\left(\tilde{\mathbf{U}}_{r} \mathbf{R}_{\mathbf{b}_{r}} \tilde{\mathbf{U}}_{r}^{H}+\tau_{r} \mathbf{I}\right)^{-1}=\prod_{k=1}^{p_{r}} \frac{1}{d_{r, k}+\tau_{r}}\left(\frac{1}{\tau_{r}}\right)^{N-p_{r}} .
$$


The likelihood function under $H_{1}$ can thus be written as

$$
\begin{aligned}
f_{1}(\mathbf{Z})= & \frac{1}{\pi^{N L}} \prod_{k=1, r=1}^{p_{r}, L} \frac{1}{d_{r, k}+\tau_{r}}\left(\frac{1}{\tau_{r}}\right)^{N-p_{r}} \\
& \times \exp \left(-\sum_{k=1, r=1}^{p_{r}, L} \frac{\tilde{\mathbf{z}}_{r}^{H} \mathbf{P}_{\tilde{\mathbf{g}}_{r, k}} \tilde{\mathbf{z}}_{r}}{d_{r, k}+\tau_{r}}-\sum_{r=1}^{L} \frac{\tilde{\mathbf{z}}_{r}^{H} \mathbf{P}_{\tilde{\mathbf{G}}_{r}^{\perp}} \tilde{\mathbf{z}}_{r}}{\tau_{r}}\right) .
\end{aligned}
$$

As a result, the log-likelihood function under $H_{1}$ becomes

$$
\begin{aligned}
l_{1}(\mathbf{Z})= & -N L \ln \pi-N \sum_{r=1}^{L} \ln \tau_{r}-\sum_{k=1, r=1}^{p_{r}, L} \ln \frac{d_{r, k}+\tau_{r}}{\tau_{r}} \\
& -\sum_{k=1, r=1}^{p_{r}, L} \frac{\tilde{\mathbf{z}}_{r}^{H} \mathbf{P}_{\tilde{\mathbf{g}}_{r, k}} \tilde{\mathbf{z}}_{r}}{d_{r, k}+\tau_{r}}-\sum_{r=1}^{L} \frac{\tilde{\mathbf{z}}_{r}^{H} \mathbf{P}_{\tilde{\mathbf{G}}_{r}^{r}} \tilde{\mathbf{z}}_{r}}{\tau_{r}} .
\end{aligned}
$$

The ML-estimates of $\tau_{r}$ and of $d_{r, k}+\tau_{r}$ are obtained by maximizing this function and are given by

$$
\begin{array}{r}
\hat{\tau}_{r \mid H 1}=\frac{\tilde{\mathbf{z}}_{r}^{H} \mathbf{P}_{\tilde{\mathbf{G}}_{r}} \tilde{\mathbf{z}}_{r}}{N-p_{r}} \\
d_{r, k}+\tau_{r}=\tilde{\mathbf{z}}_{r}^{H} \mathbf{P}_{\tilde{\mathbf{g}}_{r, k}} \tilde{\mathbf{z}}_{r} .
\end{array}
$$

This implies that

$$
\begin{aligned}
l_{1}(\mathbf{Z})= & -N L \ln \pi-N \sum_{r=1}^{L} \ln \left(\frac{\tilde{\mathbf{z}}_{r}^{H} \mathbf{P}_{\tilde{\mathbf{G}}_{r}} \tilde{\mathbf{z}}_{r}}{N-p_{r}}\right) \\
& -\sum_{k=1, r=1}^{p_{r}, L} \ln \left(\left(N-p_{r}\right) \frac{\tilde{\mathbf{z}}_{r}^{H} \mathbf{P}_{\tilde{\mathbf{g}}_{r, k}} \tilde{\mathbf{z}}_{r}}{\tilde{\mathbf{z}}_{r}^{H} \mathbf{P}_{\tilde{\mathbf{G}}_{r}^{\perp}} \tilde{\mathbf{z}}_{r}}\right)-N L .
\end{aligned}
$$

Under $H_{0}$, the log-likelihood is

$$
l_{0}(\mathbf{Z})=-N L \ln \pi-N \sum_{r=1}^{L} \ln \tau_{r}-\sum_{r=1}^{L} \frac{\tilde{\mathbf{z}}_{r}^{H} \tilde{\mathbf{z}}_{r}}{\tau_{r}}
$$

and with the ML-estimate of $\hat{\tau}_{r \mid H_{0}}=\tilde{\mathbf{z}}_{r}^{H} \tilde{\mathbf{z}}_{r} / N$ under $H_{0}$, obtained by maximizing previous function, the log-likelihood under $H_{0}$ is

$$
l_{0}(\mathbf{Z})=-N L \ln \pi-N \sum_{r=1}^{L} \ln \frac{\tilde{\mathbf{z}}_{r}^{H} \tilde{\mathbf{z}}_{r}}{N}-N L .
$$

Thus with (52) and (54), it is straightforward to show that the log-likelihood ratio is written as

$$
\begin{aligned}
\ln \Lambda_{\mathrm{GSM}}(\mathbf{Z})= & -N L \ln N \\
& -\sum_{k=1, r=1}^{p_{r}, L} \ln \left(\left(N-p_{r}\right) \frac{\tilde{\mathbf{z}}_{r}^{H} \mathbf{P}_{\tilde{\mathbf{z}}_{r}, k} \tilde{\mathbf{z}}_{r}}{\tilde{\mathbf{P}}_{\tilde{\mathbf{G}}_{r}^{r}} \tilde{\mathbf{z}}_{r}}\right) \\
& +N \sum_{r=1}^{L} \ln \left(1+\frac{\tilde{\mathbf{z}}_{r}^{H} \mathbf{P}_{\tilde{\mathbf{G}}_{r}} \tilde{\mathbf{z}}_{r}}{\tilde{\mathbf{z}}_{r}^{H} \mathbf{P}_{\tilde{\mathbf{G}}_{r}} \tilde{\mathbf{z}}_{r}}\right) .
\end{aligned}
$$

We now define $h_{r, k}=\left(N-p_{r}\right) \tilde{\mathbf{z}}_{r}^{H} \mathbf{P}_{\tilde{\mathbf{g}}_{r, k}} \tilde{\mathbf{z}}_{r} / \tilde{\mathbf{z}}_{r}^{H} \mathbf{P}_{\tilde{\mathbf{G}}_{r}^{\dagger}} \tilde{\mathbf{z}}_{r}$. Then $h_{r, k}$ appears as the signal-to-noise ratio resolved onto the one-dimensional subspace $\tilde{\mathbf{g}}_{r, k}$ and the signal-to-noise ratio resolved onto the $p_{r}$-dimensional subspace is $H_{r}=\sum_{k=1}^{p_{r}} h_{r, k}=$ $\left(N-p_{r}\right) \tilde{\mathbf{z}}_{r}^{H} \mathbf{P}_{\tilde{\mathbf{G}}_{r}} \tilde{\mathbf{z}}_{r} / \tilde{\mathbf{z}}_{r}^{H} \mathbf{P}_{\tilde{\mathbf{G}}_{r}^{I}} \tilde{\mathbf{z}}_{r}$. The next step of the derivation of the GSM-GLRT is then the maximization with respect to $h_{r, k}, k=1 \ldots p_{r}$. McWhorter in [46] and Jin in [17], [47] showed that the maximization of the likelihood under $H_{1}$ with respect to $\tilde{\mathbf{g}}_{r, k}, k=1 \ldots p_{r}$ is obtained when

$$
\max \prod_{k=1}^{p_{r}} \frac{1}{h_{r, k}}=\min \prod_{k=1}^{p_{r}} h_{r, k}=H_{r}-\left(p_{r}-1\right) .
$$

With the equality $\mathbf{P}_{\tilde{\mathbf{G}}_{r}}=\mathbf{P}_{\tilde{\mathbf{U}}_{r}}$, the GSM-GLRT can thus be rewritten as

$$
\begin{aligned}
\ln \Lambda_{\mathrm{GSM}}(\mathbf{Z})= & -N L \ln N \\
& -\sum_{r=1}^{L} \ln \left(\left(N-p_{r}\right) \frac{\tilde{\mathbf{z}}_{r}^{H} \mathbf{P}_{\tilde{\mathbf{U}}_{r}} \tilde{\mathbf{z}}_{r}}{\tilde{\mathbf{z}}_{r}^{H} \mathbf{P}_{\tilde{\mathbf{U}}_{r}^{\perp}} \tilde{\mathbf{z}}_{r}}-\left(p_{r}-1\right)\right) \\
& +N \sum_{r=1}^{L} \ln \left(1+\frac{\tilde{\mathbf{z}}_{r}^{H} \mathbf{P}_{\tilde{\mathbf{U}}_{r}} \tilde{\mathbf{z}}_{r}}{\tilde{\mathbf{z}}_{r}^{H} \mathbf{P}_{\tilde{\mathbf{U}}_{r}^{\perp}} \tilde{\mathbf{z}}_{r}}\right) .
\end{aligned}
$$

\section{REFERENCES}

[1] Wehner, D. R.

High Resolution Radar.

Boston: Artech House, 1987.

[2] Van Trees, $\mathrm{H}$.

Detection, Estimation, and Modulation Theory, vol. 3. New York: Wiley, 1971.

[3] Steiner, M.

On the detection of ultrawideband radar signals. Naval Research Laboratory, Wasington, D.C., Technical Report 92-9514, 1992.

[4] Hughes, P.

A high resolution radar detection strategy. IEEE Transactions on Aerospace and Electronic Systems, AES-19 (Sept. 1983), 663-667.

[5] Rose, G.

A look at automatic detection algorithms for a X-band radar using a high resolution search mode.

Technology Service Corporation, Technical Report TSC-W75-65/kks, 1987.

[6] Gerlach, K., Steiner, M., and Lin, F. Detection of spatially distributed targets in white noise. IEEE Signal Processing Letters, 4, 7 (July 1997), 198-200.

[7] Farina, A., and Russo, A.

Radar detection of correlated targets in clutter. IEEE Transactions on Aerospace and Electronic Systems, AES-22 (Sept. 1986), 513-532.

[8] Farina, A., and Studer, F.

Detection with high resolution radar: Advanced topics and potential applications.

Chinese Journal of Systems Engineering and Electronics, $\mathbf{3}$, 1 (1992), 32-34. 
[9] Kelley, E., and Forsythe, K.

Adaptive detection and parameter estimation for multidimensional signal models.

Lincoln Lab, MIT, Lexington, Technical Report 848, 1989.

[10] Burgess, K., and Van Veen, B.

Subspace-based adaptive generalized likelihood ratio detection.

IEEE Transactions on Signal Processing, 44 (Apr. 1996), 912-927.

[11] Bose, S., and Steinhardt, A.

Adaptive array detection of uncertain rank one waveforms.

IEEE Transactions on Signal Processing, 44 (Nov. 1996), 2801-2809.

[12] Kelly, E.

An adaptive detection algorithm.

IEEE Transactions on Aerospace and Electronic

Systems, AES-22 (Mar. 1986), 115-127.

[13] Gerlach, K., and Steiner, M.

Adaptive detection of range distributed targets.

IEEE Transactions on Signal Processing, 47 (July 1999), 1844-1851.

[14] Gerlach, K., and Steiner, M.

Fast converging adaptive detection of Doppler-shifted range-distributed targets.

IEEE Transactions on Signal Processing, 48 (Sept. 2000), 2686-2690.

[15] Conte, E., De Maio, A., and Ricci, G.

GLRT-based adaptive detection algorithms for range-spread targets.

IEEE Transactions on Signal Processing, 49, 7 (July 2001), 1336-1348.

[16] Robey, F., Fuhrmann, D., Kelly, E., and Nitzberg, R.

A CFAR adaptive matched filter detector.

IEEE Aerospace and Electronic Systems Magazine, 28, 1

(Jan. 1992), 208-216.

[17] Jin, Y., and Friedlander, B.

A CFAR adaptive subspace detector for second order Gaussian signals.

IEEE Transactions on Signal Processing, 53, 15 (Mar. 2005), 871-884.

[18] Conte, E., and Longo, $\mathrm{M}$.

Characterisation of radar clutter as a spherically invariant random process.

IEE Proceedings, Pt. F, 134, 2 (1987), 191-197.

[19] Ward, K.

Compound representation of high resolution sea clutter. Electronic Letters, (1981), 561-563.

[20] Gerlach, K.

Spatially distributed target detection in non-Gaussian

clutter.

IEEE Transactions on Aerospace and Electronic

Systems, 35, 3 (July 1999), 926-934.

[21] Conte, E., De Maio, A., and Ricci, G.

CFAR detection of distributed targets in non-Gaussian disturbance.

IEEE Transactions on Aerospace and Electronic

Systems, 38, 2 (Apr. 2002), 612-621.

[22] Farina, A., Scannapieco, F., and Vinelli, F.

Target detection and classification with very high range resolution radar.

In Proceedings of the International Conference on Radar, Versailles, France, Apr. 1989, 20-25.
[23] Farina, A., and Studer, F.

Detection with high resolution radar: Great promise, big challenge.

Microwave Journal, (May 1992), 32-34.

[24] Farina, A., Studer, F., and Vitiello, R.

High resolution radar for enhanced target detection. In Proceedings of the International Conference (Radar'92), Oct. 1992, 163-166.

[25] Swerling, P

Probability of detection for fluctuating targets. IEEE Transactions on Information Theory, 6, 2 (Apr. 1960), 269-308.

[26] Nakagami, M.

The m-distribution, a general formula of intensity

distriubtion of rapid fading.

Oxford, England: Pergamon, 1960.

[27] Gini, F., and Farina, A.

Vector subspace detection in compound-Gaussian clutter Part I: Survey and new results.

IEEE Aerospace and Electronic Systems Magazine, 38, 4 (Oct. 2002), 1295-1311.

[28] Gini, F., Greco, M., and Verrazzani, L.

Detection problem in mixed clutter environment as Gaussian problem by adaptive pre-processing. Electronic Letters, 31, 14 (July 1995), 1189-1190.

[29] Gini, F.

A cumulant-based adaptive technique for coherent radar detection in a mixture of $\mathrm{K}$-distributed clutter and Gaussian disturbance.

IEEE Transactions on Signal Processing, 45, 6 (June 1997), 1507-1519.

[30] Gini, F., Greco, M., and Farina, A.

Clairvoyant and adaptive signal detection in non-Gaussian clutter: A data-dependent threshold interpretation.

IEEE Transactions on Signal Processing, 47, 6 (June 1999), 1522-1531.

[31] Pulsone, N., and Raghavan, R. Analysis of an adaptive CFAR detector in non-Gaussian interference.

IEEE Transactions on Aerospace and Electronic Systems, 35, 3 (July 1999), 903-916.

[32] Rangaswamy, M., and Michels, J.

Adaptive signal processing in non-Gaussian noise backgrounds.

In Proceedings of the 9th IEEE-SSAP Workshop, Portland, OR, Sept. 1998.

[33] Rangaswamy, M., Michels, J., and Weiner, D. Multichannel detection algorithm for correlated non-Gaussian random processes based on innovations. IEEE Transactions on Signal Processing, 43, 8 (Aug. 1995), 1915-1922.

[34] Conte, E., Lops, M., and Ricci, G.

Adaptive detection schemes in compound-Gaussian clutter.

IEEE Transactions on Aerospace and Electronic Systems, 34, 4 (Oct. 1998), 1058-1069.

[35] Gini, F., and Greco, M. Suboptimum approach for adaptive coherent radar detection in compound-Gaussian clutter. IEEE Transactions on Aerospace and Electronic Systems, 35, 3 (July 1999), 1095-1104. 
Gini, F., and Michels, J.

Performance analysis of two covarariance matrix estimators in compound-Gaussian clutter.

IEE Proceedings, Pt. F, 146, 3 (June 1999), 133-140.

[37] Gini, F., and Greco, M.

Covariance matrix estimation for CFAR detection in correlated heavy-tailed clutter.

Signal Processing (special section on Signal Processing with Heavy Tailed Distributions), 2002.

[38] Conte, E., De Maio, A., Farina, A., and Foglia, G. Training data selection for adaptive radar. In Proceedings of International Conference on Radar Systems, 2004.

[39] Keller, J.

Geometric theory of diffraction.

Journal of the Optical Society of America, (Jan. 1962), 116-130.

[40] Kouyoumjian, R., and Pathak, P.

A uniform geometrical theory of diffraction for an edge in a perfectly conducting surface.

In Proceedings of the IEEE, 62 (Nov. 1974), 1448-1461.

[41] Backman, C.

Some recent developments in RCS measurements techniques.

In Proceedings of the IEEE, (Aug. 1965), 962-972.

[42] Li, Q., Rothwell, E., Chen, K., and Nyquist, D.

Scattering center analysis of radar targets using fitting scheme and genetic algorithm.

IEEE Transactions on Antennas Propagation, 44 (1996), 198-207.

[43] Moon, T., and Bawden, P.

High resolution RCS measurements of boats.

Proceedings of the IEE, Pt. F, 138, 3 (June 1991), 218-222.

[44] Gini, F.

Sub-optimum coherent radar detection in a mixture of K-distributed and Gaussian clutter.

IEE Proceedings, Pt. F, 144, 1 (Feb. 1997), 39-48.

[45] Sangston, K., Gini, F., Greco, M., and Farina, A.

Structures for radar detection in compound Gaussian

clutter.

IEEE Transactions on Aerospace and Electronic

Systems, 35, 2 (Apr. 1999), 445-458.

[46] McWhorter, L. T., and Scharf, L. L.

Matched subspace detectors for stochastic signals.

In 12th MIT Lincoln Labs Workshop on Adaptive Sensor and array Processing, Lexington, MA, Mar. 2003.

[47] Jin, Y., and Friedlander, B.

Detection of distributed sources using sensor arrays.

IEEE Transactions on Signal Processing, 52, 6 (June 2004), 1537-1548.

[48] Lavielle, M., Moulines, E., and Cardoso, J.

A maximum likelihood solution to DOA estimation for discrete sources.

In Proceedings of the 7th Workshop on Statistical Signal and Array Processing, Quebec City, Canada, 1994.

[49] Chung, P., and Böhme, J.

DOA estimation using fast EM and SAGE algorithms.

Signal Processing, 82 (2002), 1753-1762.

[50] Chung, P., Böhme, J., and Hero, A.

Tracking of multiple moving sources using recursive EM algorithm.

EURASIP Journal on Applied Signal Processing, 18

(2004), 1-11.
[51] Çekli, E., and Çirpan, H.

Unconditional maximum likelihood approach for localization of near-field sources: Algorithm and performances analysis.

AEU International Journal of Electronics and

Communications, 57, 1 (2003), 9-15.

[52] Snyder, D., O'Sullivan, J. A., and Miller, M. I.

The use of maximum likelihood estimation for forming images of diffuse radar targets from delay-Doppler data. IEEE Transactions on Information Theory, 35, 3 (1989), 536-548.

[53] Lanterman, A.

Statistical radar imaging of diffuse and specular targets using an expectation-maximization algorithm.

In E. G. Zelnio (Ed.), Proceedings of SPIE, Algorithms for Synthetic Aperture Radar Imagery VII, vol. 4053, Orlando, FL, Apr. 2000, 20-31.

[54] Schmidt, R.

Multiple emitter location and signal paramter estimation. IEEE Transactions on Antennas Propagation, 34 (1986), 276-280.

[55] Paulraj, R. R., and Kailath, K. T.

ESPRIT-A subspace rotation approach to esimation of parameters of cisoids in noise.

IEEE Transactions on Acoustics, Speech, and Signal

Processing, 34 (1986), 1340-1342.

[56] Akaike, $\mathrm{H}$.

A new look at the statistical model identification. IEEE Transactions on Automatic Control, AC-19, 6 (Dec. 1974), 716-723.

[57] Rissanen, J.

Modeling by shortest data description length. Automatica, 14 (1978), 465-471.

[58] Gini, F., and Bordoni, F.

On the behaviour of information theoretic criteria for model order selection of InSar signals corrupted by multiplicative noise.

Signal Processing, 83 (2003), 1047-1063.

[59] Scharf, L.

Statistical Signal Processing. Reading, MA: Addison-Wesley, 1991.

[60] Ward, K., Baker, C., and Watts, S.

Maritime surveillance radar. Part 1: Radar scattering from the ocean surface.

IEE Proceedings, Pt. F, 1990, 51-61.

[61] Watts, S., and Wards, K.

Spatial correlation in K-distributed sea clutter.

IEE Proceedings, Pt. F, 134 (1987), 526-532.

[62] Conte, E., Lops, M., and Ricci, G.

Asymptotically optimum radar detection in compound

Gaussian clutter.

IEEE Transactions on Aerospace and Electronic

Systems, 31, 2 (Apr. 1995), 617-625.

[63] Tough, R., and Ward, K.

The correlation properties of gamma and other non-Gaussian processes generated by memoryless non-linear transformation.

Journal of Physics D: Applied Physics, 1999, 3075-3084.

[64] Stoica, P., and Söderström, T.

Statistical analysis of MUSIC and subspace rotation estimates of sinusoidal frequencies.

IEEE Transactions on Signal Processing, 39, 8 (Aug. 1991), 1836-1847. 
[65]

DeGraaf, S.

SAR imaging via modern 2-D spectral estimation methods.

IEEE Transactions on Signal Processing, 7, 5 (May 1998).

[66] Radoï, E.

Contribution à la reconnaissance des objets 3D à partir de leur signature électromagnétique.

Ph.D. dissertation, Université de Bretagne Occidentale, 1999.

[67] Blum, R., and McDonald, K.

Analysis of STAP algorithms for cases with mismatched steering and clutter statistics.

IEEE Transactions on Signal Processing, 48 (Feb. 2000),

301-310.
[68] De Maio, A.

Robust adaptive radar detection in the presence of steering vector mismatches.

IEEE Transactions on Aerospace and Electronic Systems, 41, 4 (Oct. 2005), 1322-1337.

[69] Stoica, P., and Söderström, T.

On a novel subspace-based approach to parameter estimation.

Digital Signal Processing: Rev. J, 5, 4 (Oct. 1995), 237-242. 

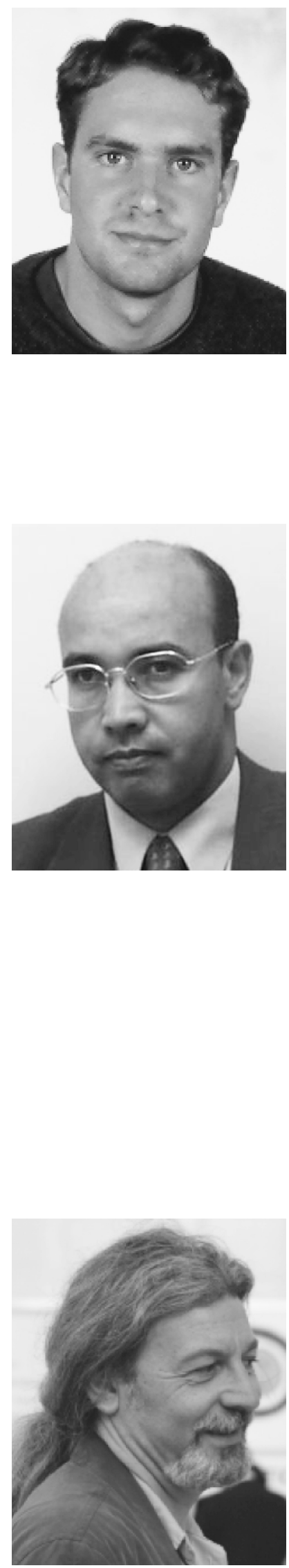

Nicolas Bon was born in Lannion, France, on February 9, 1979. He received his M.S. degree in electronic engineering form the University of Brest, France, in 2003 and graduated from the engineering school ENSIETA (Ecole Nationale Supérieure des Ingénieurs des Etudes et Techniques d'Armement) of Brest, France, in 2003.

$\mathrm{He}$ is currently with the laboratory E3I2-EA3876, ENSIETA and Thales Airborne Systems, Brest. His main research interests include signal processing, spectral estimation, radar imaging, target detection and tracking as well as automatic target recognition.

Ali Khenchaf received his masters degree in statistical data processing from the University of Rennes I, in 1989. In 1992, he received his Ph.D degree in electronic systems and computer networks from the University of Nantes.

From 1989 until 1993, he was a researcher at IRCCyN (Institut de Recherche en Communications et Cybernetique de Nantes, UMR CNRS 6597) Laboratory which is a joined laboratory between EPUN (Ecole polytechnique de l'Université de Nantes, France) and ECN (Ecole Centrale de Nantes). His researches and teaching courses are in the fields of numerical mathematics, electromagnetic wave propagation, waves \& microwaves, signal processing and operational research theory. From 1993 to 2001, he held an assistant professor position at the University of Nantes. Since September 2001, he has been with the ENSIETA (Ecole Nationale Supérieure des Ingénieurs des Etudes et Techniques d'Armement), where he is now a professor and is the head of laboratory E3I2-EA3876. His research interests include radar waves scattering, microwave remote sensing, electromagnetic wave propagation, scattering in random media, bistatic scattering of electromagnetic waves and target parameters estimation.

René Garello (M'85-SM'96-F'06) was born in 1953. He received the Ph.D. degree in signal processing at the Institut National Polytechnique de Grenoble (INPG) in 1981.

From 1982 to 1984 he worked as a research associate at Aeronomy Lab, National Oceanic and Atmospheric Administration (NOAA) at Boulder, CO. He joined the Ecole Nationale Supérieure des Télécommunications de Bretagne (ENST Bretagne), Brest, France in 1985. Since 1988 he has been professor in this engineering school in the field of signal processing and image processing. His main research interests lie in remote sensing, two-dimensional signal processing, statistical and spectral analysis applied to ocean surface features detection and characterization.

Dr. Garello started a French IEEE/OES chapter in 1993 of which he became chairman in 1995. Since 1993 he has been involved in the organization of the Student Poster Competition for the OCEANS' series of conferences. For OCEANS'98, held in Nice, France, he was Cochair of the Technical Program Committee, Chair of the Publicity Committee, and Chair of the Student Poster Competition. Since 1997, he has been associate editor for Region 8 of the IEEE Journal of Oceanic Engineering and he was elected to the OES Adcom Committee in 1998, 2002, and 2005. He was the General Chairman of the IEEE/OES Oceans'05 Europe Conference and Exhibition, June 20-23, 2005. He was elected Vice-President Conference Operations for the IEEE/OES in 2005. He is also an active member of the IEEE Geoscience and Remote Sensing Society. 